\title{
Instability wave models for the near-field fluctuations of turbulent jets
}

\author{
K. Gudmundsson $\$$ and Tim Colonius \\ Division of Engineering and Applied Science, California Institute of Technology, Pasadena, \\ CA 91125, USA
}

(Received 16 November 2010; revised 2 August 2011; accepted 17 September 2011; first published online 15 November 2011)

Previous work has shown that aspects of the evolution of large-scale structures, particularly in forced and transitional mixing layers and jets, can be described by linear and nonlinear stability theories. However, questions persist as to the choice of the basic (steady) flow field to perturb, and the extent to which disturbances in natural (unforced), initially turbulent jets may be modelled with the theory. For unforced jets, identification is made difficult by the lack of a phase reference that would permit a portion of the signal associated with the instability wave to be isolated from other, uncorrelated fluctuations. In this paper, we investigate the extent to which pressure and velocity fluctuations in subsonic, turbulent round jets can be described as linear perturbations to the mean flow field. The disturbances are expanded about the experimentally measured jet mean flow field, and evolved using linear parabolized stability equations (PSE) that account, in an approximate way, for the weakly nonparallel jet mean flow field. We utilize data from an extensive microphone array that measures pressure fluctuations just outside the jet shear layer to show that, up to an unknown initial disturbance spectrum, the phase, wavelength, and amplitude envelope of convecting wavepackets agree well with PSE solutions at frequencies and azimuthal wavenumbers that can be accurately measured with the array. We next apply the proper orthogonal decomposition to near-field velocity fluctuations measured with particle image velocimetry, and show that the structure of the most energetic modes is also similar to eigenfunctions from the linear theory. Importantly, the amplitudes of the modes inferred from the velocity fluctuations are in reasonable agreement with those identified from the microphone array. The results therefore suggest that, to predict, with reasonable accuracy, the evolution of the largest-scale structures that comprise the most energetic portion of the turbulent spectrum of natural jets, nonlinear effects need only be indirectly accounted for by considering perturbations to the mean turbulent flow field, while neglecting any non-zero frequency disturbance interactions.

Key words: absolute/convective instability, hydrodynamic noise, jet noise

\section{Introduction}

Prior to the 1970s, the prevailing view of the turbulence in free shear flows was one of an agglomeration of incoherent, fine-scale fluctuations. This changed with the

$\dagger$ Email address for correspondence: k.gudmundsson@utwente.nl

$\ddagger$ Present address: University of Twente, 7522 NB Enschede, The Netherlands. 
experimental observations of Crow \& Champagne (1971), Brown \& Roshko (1974) and Winant \& Browand (1974), who reported findings of coherent structures in both jet and planar mixing-layer turbulence. In an initially laminar shear layer, these fluctuations initially grow in amplitude by receiving energy from the inflectional mean velocity profile via the Kelvin-Helmholtz instability. Further downstream, nonlinear interactions provide for saturation, pairings and other dynamics, before the wave at a given frequency ultimately decays.

While large-scale coherent structures qualitatively reminiscent of instability waves have also been observed in turbulent jets (Brown \& Roshko 1974; Michalke \& Fuchs 1975; Maestrello \& Fung 1979; Morris, Giridharan \& Lilley 1990; Arndt, Long \& Glauser 1997; Pinier, Hall \& Glauser 2006; Suzuki \& Colonius 2006; Tinney \& Jordan 2008), it is difficult to assert whether they can be quantitatively identified with instability waves. One essential difficulty is the acquisition of data with which to compare theory. In unforced jets, large-scale structures are intermittent and accompanied by smaller-scale, less coherent fluctuations, making their unambiguous detection challenging. In general, one expects only a portion of the fluctuating flow field to be associated with instability waves. The situation is further confounded at higher Reynolds numbers $R e$, where the relative energy associated with largescale coherent structures is diminished due to increased production of smaller-scale turbulence.

One way to overcome this ambiguity is to provide artificial harmonic disturbances near the nozzle exit, which in turn provides a phase reference with which to correlate (phase-average) measured fluctuating velocities and pressures. This approach has proved successful in associating the forced large-scale structures with frequencies and eigenfunctions obtained from linear stability analysis (Mattingly \& Chang 1974; Moore 1977; Zaman \& Hussain 1980; Mankbadi \& Liu 1981; Strange \& Crighton 1983; Mankbadi 1985; Tam \& Morris 1985; Tanna \& Ahuja 1985; Cohen \& Wygnanski 1987; Petersen \& Samet 1988). Despite successes, questions persist as to the extent to which the theory can be applied to natural (unforced) jets, which is the topic of this paper.

A different approach applicable to natural turbulent jets was developed by Suzuki \& Colonius (2006, hereafter referred to as SC). They noted that pressure fluctuations associated with instability waves with subsonic convection speeds take the form of an evanescent wave field (or pseudo-sound) in the region just outside the jet shear layer where fluctuation levels are sufficiently small that linearization is appropriate. Their hypothesis is that, with sufficient spatial resolution, an array of microphones placed in this region would therefore better segregate between that portion of the pressure field associated with instability waves and that portion arising from uncorrelated, smaller-scale turbulent fluctuations.

This idea is illustrated in figure 1, which shows a cartoon of the radial decay of pressure fluctuations in a turbulent jet. The figure shows two cross-over regions. The first cross-over is from a region dominated by nonlinear fluctuation levels to an outer, linear region, where pressure levels fall off exponentially with radius. These regions are collectively referred to as the hydrodynamic regime, alluding to the largely convective character of the resident fluctuations. The next cross-over is from the linear hydrodynamic to the acoustic region in which pressure fluctuations propagate at the ambient speed of sound while decaying in inverse proportion to radius. It should be noted that the seemingly organized wavepacket structure of the nearjet pressure field had been appreciated since the early days of jet noise research 


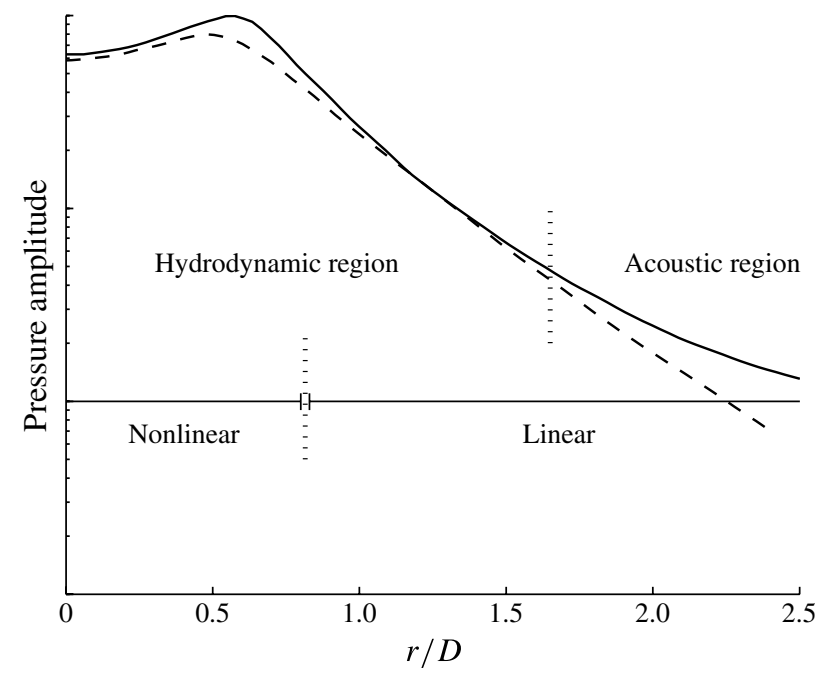

FIGURE 1. Radial distribution of pressure fluctuations of the axisymmetric mode in a turbulent jet. Shown is a hypothetical r.m.s. pressure distribution (full curve), along with a locally parallel eigenfunction from linear stability analysis (dashed curve). Indicated are the regions demarcated by type of dominating pressure fluctuations: nonlinear/linear hydrodynamic (evanescent) and acoustic.

(Mollo-Christensen 1967), but the first quantitative comparisons of the pressure measured in this region with linear stability theory (LST) was provided by SC.

Using the caged microphone array described in detail in $\S 3.2$, SC used a beamforming algorithm to identify the signatures of convecting wavepackets, and compared the measurements with predictions of a locally parallel linear stability model that used the jet mean flow field as the base flow. They obtained good agreement for the phase speed and spatial evolution near the most unstable frequency, and upstream of the end of the potential core, but the agreement at sub-peak frequencies and further downstream was less favourable. It should be noted that the linear theory cannot predict the overall amplitude of the structure - the comparisons were made by choosing a constant that gave the best fit between theory and measurement at each frequency and azimuthal wavenumber.

Here we extend the approach of SC in two significant ways. First, we employ parabolized stability equations (PSE), introduced by Bertolotti \& Herbert (1991). This approach represents a refinement of the locally parallel approach whereby both nonparallel and nonlinear effects can be retained in the analysis of slowly spreading, convectively unstable flows such as boundary layers (Bertolotti \& Herbert 1991; Bertolotti, Herbert \& Spalart 1992; Chang et al. 1993), planar mixing layers (Day, Mansour \& Reynolds 2001; Cheung \& Lele 2009) and jets (Balakumar 1998; Yen \& Messersmith 1998; Malik \& Chang 2000; Piot et al. 2006; Gudmundsson \& Colonius 2009; Ray \& Cheung 2009). While it is computationally feasible nowadays to perform a global stability analysis of the jet mean flow field (Chomaz 2005), we prefer PSE with an eye towards developing rapidly computable, reduced-order models for the farfield sound associated with large-scale structures (Colonius, Samanta \& Gudmundsson 2010; Reba, Narayanan \& Colonius 2010).

Secondly, we address the overall amplitude of the large-scale structures and show that amplitude values inferred by matching theory and measurement along the microphone array are, to a reasonable approximation, consistent with near-field 
velocity fluctuations measured by particle image velocimetry (PIV) throughout the jet, even downstream of the close of the potential core. For both sets of measurements, we show that filtering the signals via the proper orthogonal decomposition (POD) (e.g. Lumley 1967; Arndt et al. 1997), which separates data into uncorrelated portions ordered by their energy, provides for a cleaner assessment of the theory, especially for higher frequencies and downstream of the close of the potential core. The results provide strong evidence for the proposition that, in natural, turbulent jets, nonlinear effects need only be considered in determining the mean flow field, with the evolution of large-scale instability wave structures occurring linearly, essentially independently of any non-zero frequency wave interactions. While this idea is certainly not new (e.g. Malkus 1956), we believe that the present work is the first to perform an extensive analysis of natural, high-Reynolds-number turbulent jet data from this point of view, and to show that a rather precise and consistent match between linear theory and measurements can be obtained.

The remainder of this paper is organized as follows. In $\S 2.1$ we discuss issues related to the choice of basic flow, linearization and models for fluctuations. In $\S 3$ we review the experimental techniques used to obtain the data for our study, and discuss the actual data. In $\S 4$ we discuss the Fourier and proper orthogonal decomposition of the microphone data, finally making comparisons with both the unfiltered and PODfiltered data. In $\S 5$ we analyse velocity measurements made inside the jet, making comparisons with the instability model of PSE. Finally, in $\S 6$, we summarize our conclusions and discuss the extensions of these ideas for reduced-order models of sound generation by large-scale structures.

\section{Instability wave models}

\subsection{Theoretical background}

Turbulent jets may be characterized by the time-averaged and temporal fluctuations of their flow field, $\boldsymbol{q}(\boldsymbol{x}, t)=\overline{\boldsymbol{q}}(\boldsymbol{x})+\boldsymbol{q}^{\prime}(\boldsymbol{x}, t)$, where we take $\boldsymbol{q}=\left(u_{x}, u_{r}, u_{\theta}, \rho, T\right)^{\mathrm{T}}$, which represent the axial, radial and azimuthal components of velocity, the density and the temperature, respectively. The fluctuations arise at various scales and have varying characteristics, such as degree of spatial and temporal coherence. This can be formalized by triply decomposing (Hussain \& Reynolds 1970) the flow field by separating $\boldsymbol{q}^{\prime}(\boldsymbol{x}, t)$ into coherent and incoherent fluctuations, an approach that is particularly useful in forced flows, where the coherent part can be assembled via phase averaging. As we ultimately ignore nonlinearity in our evolution model for the disturbances, we do not require the triple decomposition but note that the equations below could be derived in such a framework by ignoring the interactions between the incoherent fluctuations with the resolved, coherent ones.

Owing to homogeneity in the azimuthal direction, and assumed statistical stationarity in time, we decompose the fluctuations into frequency and azimuthal Fourier modes,

$$
\boldsymbol{q}^{\prime}(\boldsymbol{x}, t)=\sum_{m} \sum_{\omega} \tilde{\boldsymbol{q}}_{m, \omega}(x, r) \mathrm{e}^{\mathrm{i} m \theta} \mathrm{e}^{-\mathrm{i} \omega t},
$$

and insert these into the compressible Navier-Stokes equations, which for brevity we represent in operator form as $\mathscr{N}(\boldsymbol{q})=0$, to obtain

$$
\begin{gathered}
\mathscr{N}(\overline{\boldsymbol{q}})=R_{0,0}, \\
\mathscr{L}\left(\tilde{\boldsymbol{q}}_{m, \omega}\right)=R_{m, \omega} \quad \forall \omega \neq 0,
\end{gathered}
$$


where $\mathscr{L}$ represents the linearization of $\mathscr{N}(\boldsymbol{q})$ about the (time-invariant) mean flow field $\overline{\boldsymbol{q}}$; and $R_{m, \omega}$ represents the (generalized) Reynolds stresses, which involve both double and triple convolutions of the Fourier components $\tilde{\boldsymbol{q}}_{m^{\prime} \omega^{\prime}}$ over all doublets or triplets, respectively, of indices summing to $(m, \omega)$, except for those terms with $\omega^{\prime}=0$, which already appear on the left-hand sides.

Equation (2.2) represents the Reynolds-averaged Navier-Stokes (RANS) equations. In this study, we take the mean turbulent flow field as given, as determined by the experimental measurements discussed in $\S 3$. (In future modelling efforts, the mean flow could be determined by solving the RANS equations.) We further assume that (2.2) is identically satisfied by the measured mean flow field. We wish to test the hypothesis that, for unforced jets with inlet disturbances that are of essentially random phase, the mean flow distortion (including spatial amplification and decay) of disturbances inherent in the linear operator, $\mathscr{L}$, is sufficient to predict the statistics of the fluctuations, $\boldsymbol{q}^{\prime}$, while neglecting all mode-mode interactions, $R_{m, \omega}$. By statistics of the fluctuations, we mean specifically the power spectral density (PSD) of the disturbance field at frequencies appropriate to large-scale turbulent structures.

With real frequency, $\omega$, equation (2.3) with $R_{m, \omega}=0$ represents a boundary-value problem with a (generally unknown) disturbance amplitude, radial profile and phase at the inlet (just downstream of the jet nozzle exit). The solutions are not instability waves in the classical sense, since they are globally bounded by the imposition of boundary conditions that disturbances decay to infinity. However, for frequencies such that the mean flow field can be considered as slowly varying, disturbances will take the form of (potentially) spatially growing (Kelvin-Helmholtz) waves at a local value of $x$. We find a limited portion of the full spectrum of possible solutions by imposing the spatial wave ansatz and solve the corresponding PSE, as described more fully below. Since linear equations are being solved, we may, in principle, superpose these (approximate) solutions onto other solutions of the full equations to obtain the complete solution for given inlet boundary conditions.

However, we restrict our attention to (what we call) the instability wave solutions and attempt to search for the signatures of these structures in experimental data that, presumably, represent the complete superposition. In so doing, we note that we need not invoke the linear $\left(R_{m, \omega}=0\right)$ assumption for all components of the solution - only for those specific modes we find. In other words, provided the linear approximation is sufficiently valid for this limited portion of the disturbance spectrum, then it is irrelevant whether other disturbance solutions evolve linearly or are forced by other modes.

The question of the importance of direct nonlinear interactions of instability waves in jets has been considered before. Strange \& Crighton (1983), in studying a forced $R e_{D}=10^{4}$ jet, found radial structure and phase velocities to be well predicted by linear stability analysis, but not streamwise rates of amplification, attributing this to the nonlinear response of the instability wave to the periodic forcing conditions. Similar conclusions were drawn by Gaster, Kit \& Wygnanski (1985) in the context of a forced planar mixing layer. Finally, the results of natural and forced low-Reynolds-number jets considered by Laufer \& Yen (1983) have been interpreted in terms of nonlinear saturation and vortex pairing associated with nonlinear interactions (Huerre \& Crighton 1983; Fleury, Bailly \& Juve 2005). By contrast, the jets we study are unforced and fully turbulent. Instability growth rates associated with the mean velocity profile are small compared to those that would be obtained for a laminarly spreading jet; the resulting fluctuations therefore attain comparatively lower amplitudes. Meanwhile, the faster spread rate of the mean flow may cause disturbances to become neutral, and 
decay, before attaining amplitudes at which significant nonlinear interaction would occur. As a first step, in this study we neglect nonlinear interactions entirely, and show a posteriori that this provides for reasonable agreement with pressure and velocity measurements in fully turbulent jets, at least for that (low-frequency) portion of the full disturbance spectrum that has the form of instability waves.

\subsection{The parabolized stability equations}

Solutions of (2.3) with $R_{m, \omega}=0$ that have the form of a spatially amplifying or decaying wave may be found using a variety of approximations, from parallel or quasi-parallel spatial linear stability analysis, where the spread of the mean flow is ignored, to multiple-scale analysis (e.g. Crighton \& Gaster 1976) and PSE that account for a slowly diverging jet mean flow field. At the other extreme, one can, without parallel-flow or parabolizing approximations, directly compute solutions for particular inlet disturbances. The latter is not much less computationally intensive than large eddy simulation, and, in any event, the inlet disturbances are not in general known. Regarding the multiple-scale and PSE approaches, the latter two have been shown to result in comparable predictions in linear contexts (e.g. Chang et al. 1993) for convectively unstable flows. However, the advantages of the PSE are the computational efficiency and the relative ease with which nonlinear wave-wave interactions may be included in future. For this reason, we employ the PSE; the details of our implementation are discussed below.

The PSE (Herbert 1997) explicitly account for the effects of modest mean flow spreading. Building on ideas from multiple-scale analysis, Bertolotti \& Herbert (1991) suggested that the function $\tilde{\boldsymbol{q}}(x, r)$ be separated into a function varying streamwise at a similar rate as the mean flow and a rapidly varying function capturing the wave-like behaviour of the large-scale structure:

$$
\boldsymbol{q}^{\prime}(\boldsymbol{x}, t)=\hat{\boldsymbol{q}}(x, r) \mathrm{e}^{\mathrm{i} \int^{x} \alpha(\xi) \mathrm{d} \xi} \mathrm{e}^{\mathrm{i} m \theta} \mathrm{e}^{-\mathrm{i} \omega t} .
$$

This assumption represents the parabolization of the linearized equations and allows a marching solution, the $x$-coordinate becoming time-like. We note, however, that the resulting equations for $\hat{\boldsymbol{q}}$ (system (2.5) below) are not completely parabolic, as discussed by Li \& Malik (1996, 1997).

Substituting the decomposition given by (2.4) into the governing equations, we obtain in symbolic form the system of equations governing the evolution of the shape functions $\hat{\boldsymbol{q}}(x, r)$ :

$$
(\boldsymbol{A}(\overline{\boldsymbol{q}}, \alpha, \omega)+\boldsymbol{B}(\overline{\boldsymbol{q}})) \hat{\boldsymbol{q}}+\boldsymbol{C}(\overline{\boldsymbol{q}}) \frac{\partial \hat{\boldsymbol{q}}}{\partial x}+\boldsymbol{D}(\overline{\boldsymbol{q}}) \frac{\partial \hat{\boldsymbol{q}}}{\partial r}=\frac{1}{R e} \boldsymbol{E}(\overline{\boldsymbol{q}}) \hat{\boldsymbol{q}} .
$$

Expressions for the operators $\boldsymbol{A}$ to $\boldsymbol{E}$ may be found in Gudmundsson (2010). We discretize this system using fourth-order central differences in the radial direction, closing the domain with the characteristic boundary conditions of Thompson (1987). While previous studies (e.g. Herbert 1997) have shown boundary conditions based on asymptotic decay rates from LST to be sufficiently accurate, we find the characteristic formulation to be more robust in that it allows a smaller computational domain. The streamwise derivative is approximated via first-order implicit Euler differences. The solution $\hat{\boldsymbol{q}}_{j+1}$ at $x_{j+1}$ is then obtained from that at $x_{j}$ via the solution of

$$
\left(\Delta x\left(\boldsymbol{A}+\boldsymbol{B}+\boldsymbol{D} \frac{\mathrm{d}}{\mathrm{d} r}-\frac{1}{R e} \boldsymbol{E}\right)+\boldsymbol{C}\right)_{j+1} \hat{\boldsymbol{q}}_{j+1}=\boldsymbol{C}_{j+1} \hat{\boldsymbol{q}}_{j} .
$$




$\begin{array}{lccc}\text { Set point } & M_{\infty} & T_{j e t} / T_{\infty} & R e \\ 3 & 0.5 & 0.96 & 7 \times 10^{5} \\ 23 & 0.5 & 1.76 & 2 \times 10^{5} \\ 7 & 0.9 & 0.85 & 16 \times 10^{5} \\ 27 & 0.9 & 1.76 & 4 \times 10^{5}\end{array}$

TABLE 1. Flow conditions investigated in this study. Set points are as defined by Tanna (1977).

The decomposition in (2.4) is ambiguous in that the streamwise development of $\boldsymbol{q}^{\prime}(\boldsymbol{x}, t)$ can be absorbed into either the shape function $\hat{\boldsymbol{q}}(x, r)$ or the wavenumber/growth rate $\alpha(x)$. This ambiguity is usually resolved via the additional constraint

$$
\int_{0}^{\infty} \hat{\boldsymbol{q}} \frac{\partial \hat{\boldsymbol{q}}^{*}}{\partial x} r \mathrm{~d} r=0
$$

which removes any exponential factor from the shape functions $\hat{\boldsymbol{q}}$, ensuring their slow (algebraic) streamwise variation. This provides an algorithm for updating $\alpha^{j+1}$ :

$$
\alpha_{n+1}^{j+1}=\alpha_{n}^{j+1}-\frac{i}{\Delta x} \frac{\int_{0}^{\infty}\left(\hat{\boldsymbol{q}}_{n}^{j+1}\right)^{*}\left(\hat{\boldsymbol{q}}_{n}^{j+1}-\hat{\boldsymbol{q}}^{j}\right) r \mathrm{~d} r}{\int_{0}^{\infty}\left|\hat{\boldsymbol{q}}_{n}^{j+1}\right|^{2} r \mathrm{~d} r} .
$$

We now iterate between (2.6) and (2.8) to advance the solution from $x_{j}$ to $x_{j+1}$.

Initial conditions $(\hat{\boldsymbol{q}}, \alpha)_{x_{0}}$ optimally come from a solution that includes the local effects of flow spread. Day et al. (2001) used asymptotic expansions to this end, but found only minor benefits over the quasi-parallel LST solution based on $\overline{\boldsymbol{q}}\left(x_{0}, r\right)$. In this work we also use the LST solution as an initial condition.

\section{Experimental measurements and data processing}

We investigate two pairs of heated and cold round jets at acoustic Mach numbers $M_{\infty}=U_{\text {jet }} / a_{\infty}=0.5$ and 0.9 . The flow conditions are listed in table 1 . These jets were probed via stereo PIV and a caged microphone array, both described below.

\subsection{Velocity measurements}

Velocity data were obtained via stereo PIV measurements conducted by Bridges \& Wernet (2003) in the Small Hot Jet Acoustic Rig (SHJAR) at the NASA Glenn Research Center. Measurements were made in both the streamwise (i.e. $x-y)$ and cross-stream $(y-z)$ planes, with streamwise and cross-stream resolution of $0.04 D$ and $0.03 D$, respectively. Mean flow field surveys are shown in figure 2 . The mean flows consist of an ensemble average of 200 instantaneous snapshots taken $0.1 \mathrm{~s}$ apart. The snapshots can be considered uncorrelated, as the time between shots far exceeds the eddy pass-through time (the slowest jet travels roughly $350 \mathrm{D}$ in $0.1 \mathrm{~s}$ ). Also available was the set of 200 instantaneous cross-stream measurements, at various axial locations, for the cold $M_{\infty}=0.9$ jet; only the ensemble average was available for other conditions. These are analysed in $\S 5$. To obtain the azimuthal average and other azimuthal modes of the PIV data, we interpolate data, using cubic splines, onto a cylindrical grid, and then make a Fourier transform for each radial value of interest. Prior to the interpolation, the data must be shifted so as to place the axis of the 

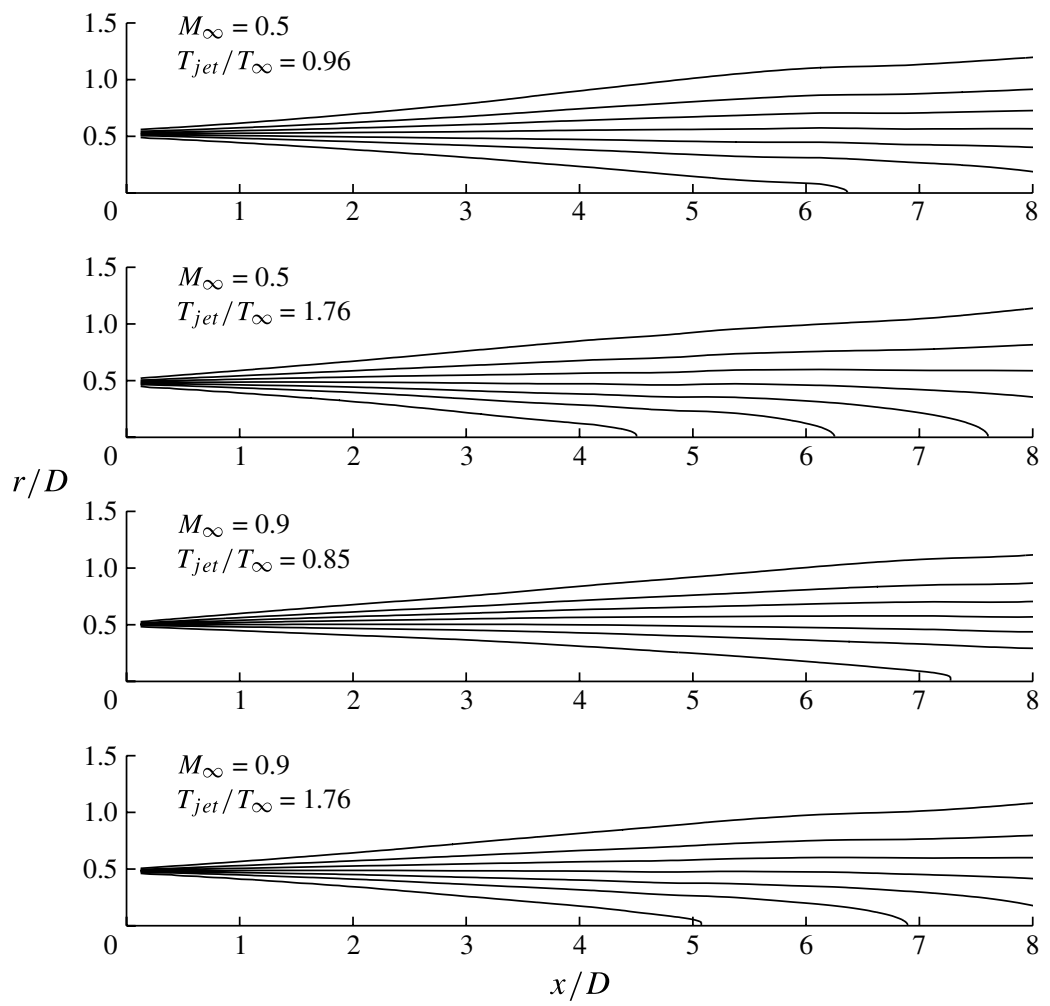

FIGURE 2. Contours of axial velocity $\bar{u}_{x} / U_{\text {jet }}$ for the four jets in table 1 . Contours are in equal increments from 0.1 to 0.99 .

cylindrical coordinate system at the geometric centre of the velocity profile $\bar{u}_{x}(x, y, z)$. We find the centre, $\left(y_{c}, z_{c}\right)$, for each $x$, via

$$
y_{c}(x)=\frac{\sum_{i} \sum_{j} y_{i} \bar{u}_{x}\left(x, y_{i}, z_{j}\right)}{\sum_{i} \sum_{j} \bar{u}_{x}\left(x, y_{i}, z_{j}\right)},
$$

and similarly for $z_{c}(x)$. A further review of the PIV camera setup, flow seeding and data processing may be found in Bridges \& Wernet (2003).

To avoid numerical issues stemming from the use of non-smooth measurements in the solution of the PSE system (2.6), we fit the PIV mean flow with a Gaussian profile similar to that used by Troutt \& McLaughlin (1982) and Tam \& Burton (1984):

$$
\frac{\bar{u}_{x}}{U_{\text {jet }}}= \begin{cases}1, & \text { if } r<R(x), \\ u_{c}(x) \exp \left(-\frac{(r-R(x))^{2}}{\delta(x)^{2}}\right), & \text { otherwise. }\end{cases}
$$

The profile parameters $R(x), \delta(x)$ and $u_{c}(x)$ are determined from the PIV data via a least-squares fitting. To ensure smooth axial variation of $\bar{u}_{x}(x, r)$, we further fit the profile parameters with a cubic polynomial, an example of which is shown in figure 3 


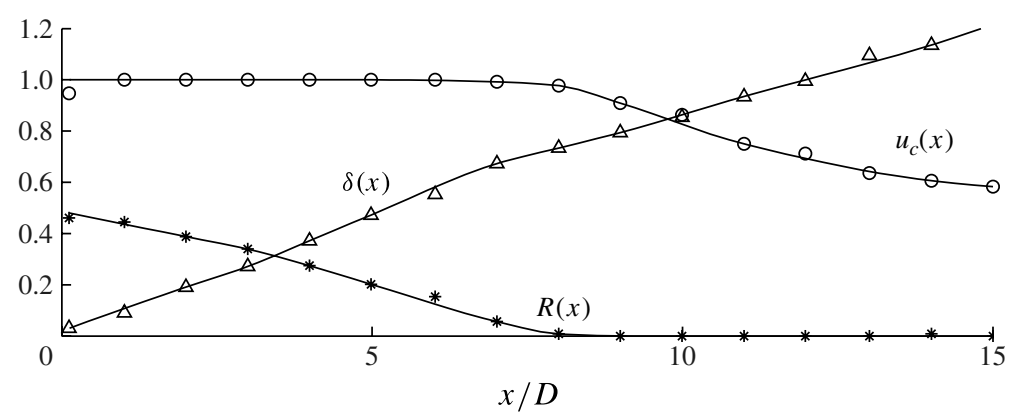

FIGURE 3. Profile parameters $R(x), \delta(x)$ and $u_{c}(x)$ as determined from PIV measurements (symbols) of the cold $M_{\infty}=0.9$ jet and fitted with a cubic polynomial (curves) to ensure smooth axial variation of $\bar{u}_{x}$.

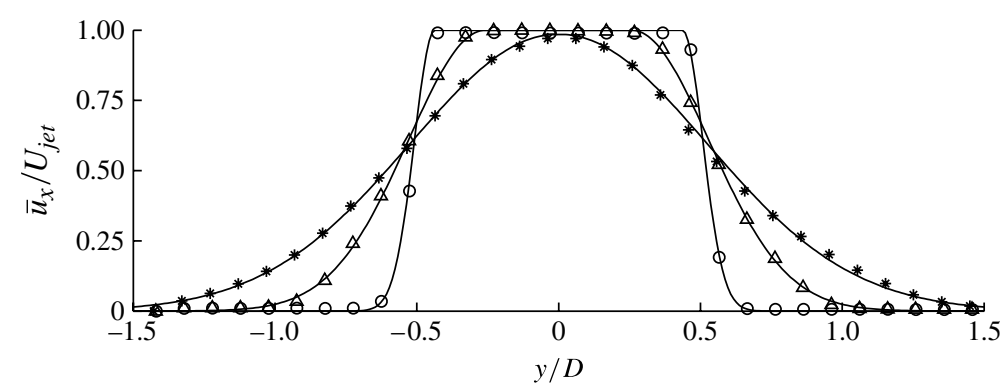

FIGURE 4. PIV measurements (symbols) of axial velocity for the cold $M_{\infty}=0.9$ jet, matched with the profile of (3.2) (curves). Shown are axial stations $x / D=1.0(0), 4.0(\triangle)$ and $8.0(*)$. Every fourth data point is shown.

for the cold $M_{\infty}=0.9$ jet. Figure 4 shows the excellent fits so obtained for the same jet; similar results are obtained at other flow conditions.

Temperature measurements were not available and were estimated from velocity via the Crocco-Busemann relation,

$$
\frac{T}{T_{\infty}}=-\frac{\bar{u}_{x}^{2}}{2}+\left(\frac{1}{\gamma-1}\left(\frac{T_{j e t}}{T_{\infty}}-1\right)+\frac{M_{\infty}^{2}}{2}\right) \frac{\bar{u}_{x}}{M_{\infty}}+\frac{1}{\gamma-1} .
$$

Transverse velocity profiles $\bar{u}_{r}(x, r)$ were, in turn, estimated from the continuity equation.

\subsection{Pressure measurements}

Pressure measurements were obtained from a caged microphone array, shown in figure 5, at the NASA Glenn SHJAR facility. This array consists of 13 concentric rings arranged on a conically expanding surface with the cone angle $\left(11.2^{\circ}\right)$ set to be slightly greater than the spread angle of the jet shear layer. The cone angle is fixed so that the relative angle between the jet and the array varies slightly with the jet operating conditions. The radius of the array was chosen in an attempt to place the microphones in the linear hydrodynamic regime, illustrated in figure 1 . Here, the pressure fluctuations are largely hydrodynamic, as discussed in the introduction. Each ring carries six microphones distributed evenly around the perimeter, allowing the resolution of the most energetic azimuthal modes, $m=0-2$. The first ring has a radius 


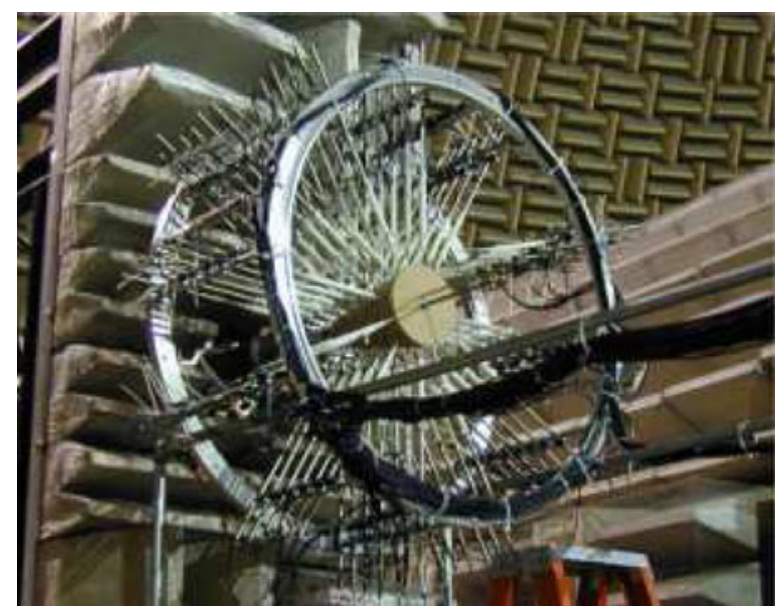

FIGURE 5. (Colour online available at journals.cambridge.org/flm) The Small Hot Jet Acoustic Rig (SHJAR) and Hydrodynamic Array at the NASA Glenn Research Center.

of $r=0.875 D$ and adjacent rings are shifted $30^{\circ}$ azimuthally and $0.625 D$ axially, for a total axial range of $8.125 \mathrm{D}$. The array is movable in the axial direction, so that the actual $x / D$ location of the rings is variable and can be gauged from the symbols representing the microphone rings in each case.

To obtain smooth PSDs, we divide the time series recorded by each microphone into 250 bins, each with a frequency resolution of $\Delta f=25 \mathrm{~Hz}$ (or $\Delta S t=\Delta f D / U_{\text {jet }}=$ 0.0043 at $M_{\infty}=0.9$, where $S t$ is the Strouhal number). The PSD is computed for each bin and the final PSD taken as the ensemble average of this set. Further details of the array design and experimental setup can be found in SC.

\subsection{Normalization of predictions}

In what follows we respectively denote measured and computed quantities with upperand lower-case characters. The time series $P(x, r, \theta, t)$ from the 78-microphone array is decomposed into its azimuthal and temporal harmonics:

$$
P(x, r, \theta, t)=\sum_{m} \int \widetilde{P}_{m, \omega}(x, r) \mathrm{e}^{-\mathrm{i} \omega t} \mathrm{~d} \omega \mathrm{e}^{\mathrm{i} m \theta} .
$$

Next, we compare $\widetilde{P}_{m, \omega}$ with the PSE prediction,

$$
\widetilde{p}_{m, \omega}(x, r)=A_{m, \omega} \hat{p}_{m, \omega}(x, r) \mathrm{e}^{\mathrm{i} \int^{x} \alpha_{m, \omega}(\xi) \mathrm{d} \xi},
$$

where $A_{m, \omega}$ represents the initial amplitude of the PSE solution $\hat{\boldsymbol{q}}$. (Note that the shape functions are first normalized at $x=x_{0}$ such that $\hat{u}_{r}$ is real-valued at $r=1 / 2$ and $\max _{r}(\hat{\boldsymbol{q}})=1$.) The system governing the evolution of $\hat{p}_{m, \omega}$ is linear and neither depends on nor predicts $A_{m, \omega}$. This we determine via amplitude matching, choosing $A_{m, \omega}$ as that minimizing the error

$$
E\left(A_{m, \omega}\right)=\sum_{j=1}^{N_{\text {ring }}}\left|\widetilde{P}_{m, \omega}^{j}-\widetilde{p}_{m, \omega}^{j}\right|^{2}
$$


where $\widetilde{P}_{m, \omega}^{j}$ and $\widetilde{p}_{m, \omega}^{j}$, respectively, denote measurements and predictions at $x=x_{j}$, and $N_{\text {ring }}$ denotes the number of microphone rings. This results in the estimate

$$
A_{m, \omega}=\frac{\sum_{j=1}^{N_{\text {ring }}}\left|I_{m, \omega}^{j} \widetilde{P}_{m, \omega}^{j}\right|}{\sum_{j=1}^{N_{\text {ring }}}\left|I_{m, \omega}^{j}\right|^{2}},
$$

where $I_{m, \omega}^{j}=\hat{p}_{m, \omega}(x, r) \mathrm{e}^{\mathrm{i} \int^{x} \alpha_{m, \omega}(\xi) \mathrm{d} \xi}$.

\section{Comparison with pressure measurements}

In this section we analyse pressure measurements made in the near field and show how PSE predictions can be significantly improved by filtering the data via the proper orthogonal decomposition.

\subsection{Pressure composition}

The pressure field of the turbulent jet at the microphone positions just outside the shear layer comprises fluctuations from various sources, both hydrodynamic and acoustic. To 'fairly' compare with the theory, which is only intended to represent the large-scale coherent structures, it is desirable to decompose the data into a correlated instability wave component and other (uncorrelated) contributions. For this purpose, we use the proper orthogonal decomposition, discussed below.

It is instructive first to consider another decomposition of the pressure, into hydrodynamic and acoustic components. While a precise, local decomposition is not possible, it is possible to decompose the signal according to the phase speed of disturbances, into those travelling at subsonic speeds (which cannot correspond to acoustic waves) and those with supersonic phase speed, which may be either acoustic or hydrodynamic, depending on their structure and propagation (if any) in the radial direction. To this end, we transform the pressure measurements along the array to obtain an axial wavenumber spectrum, $\alpha_{r}$. Analogously with Tinney \& Jordan (2008) we then reverse the transform but do so separately for wavenumbers $\alpha_{r}$ such that the phase speed is $c_{p}=\omega / \alpha_{r}<a_{\infty}$ and $c_{p} \geqslant a_{\infty}$, to obtain a decomposition

$$
P=P\left\{c_{p}<a_{\infty}\right\}+P\left\{c_{p} \geqslant a_{\infty}\right\} .
$$

This decomposition is shown in figure 6 , for the hot $M_{\infty}=0.9$ jet of this study. This figure shows contours of measured and decomposed pressure for the 13 microphones, as a function of time. The subsonic and supersonic components, respectively, have the expected slopes $\mathrm{d} x / \mathrm{d} t=0.6 U_{j e t}$ (the convective velocity) and $a_{\infty}$. Another view is given in figure 7 , where the root mean square (r.m.s.) of each component of (4.1) is shown, for each of the four flow conditions in table 1 at $m=0$. The hydrodynamic component is dominant in all cases but there is a clear trend of increasing acoustic power and thereby signal contamination with higher speed and increasing temperature. Using these data we observe that hydrodynamic and acoustic power scales approximately as $M_{\infty}^{2}$ and $M_{\infty}^{4}$, as expected from theory.

While this transform can provide an estimate of the ratio of acoustic to hydrodynamic power, it is less suited for comparing PSE predictions to the hydrodynamic component $P\left\{c_{p}<a_{\infty}\right\}$. This is due to the limited streamwise resolution and streamwise extent of our microphone array, which respectively introduce errors 
(a)

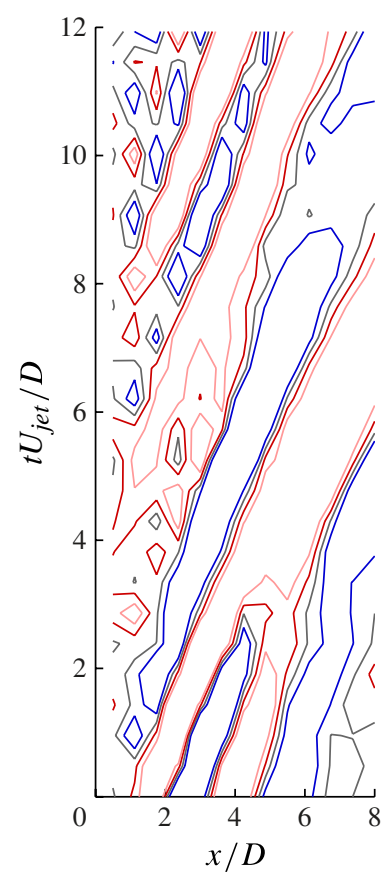

(b)

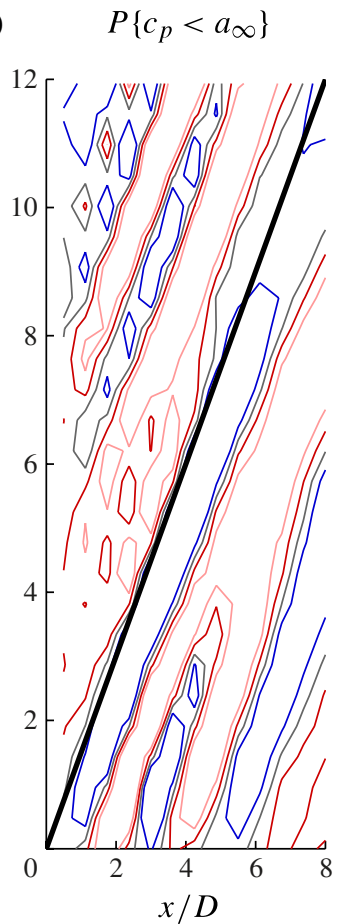

(c) $P\left\{c_{p} \geqslant a_{\infty}\right\}$

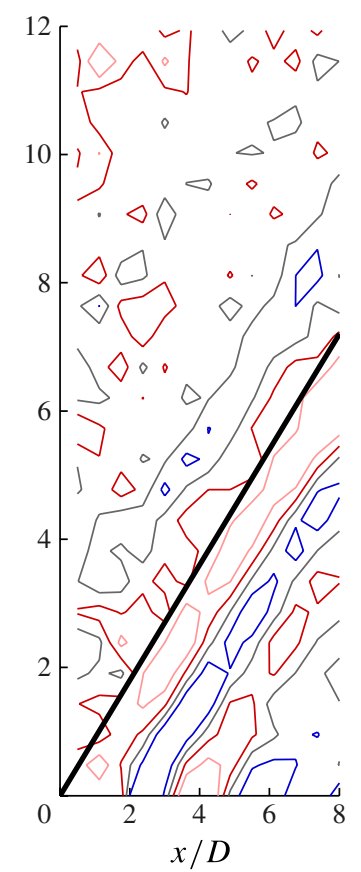

FIGURE 6. (Colour online) Instantaneous snapshots of $m=0$ perturbation pressure and its Fourier decomposition by phase speed $c_{p}$ : $(a)$ total, $(b)$ subsonic and $(c)$ supersonic pressure. The heavy lines in the subsonic and supersonic plots, respectively, have reciprocal slopes $\mathrm{d} x / \mathrm{d} t=0.6 U_{\text {jet }}$ (convective velocity) and $\mathrm{d} x / \mathrm{d} t=a_{\infty}$ (ambient speed of sound). These data are taken from measurements of the hot $M_{\infty}=0.9$ jet.
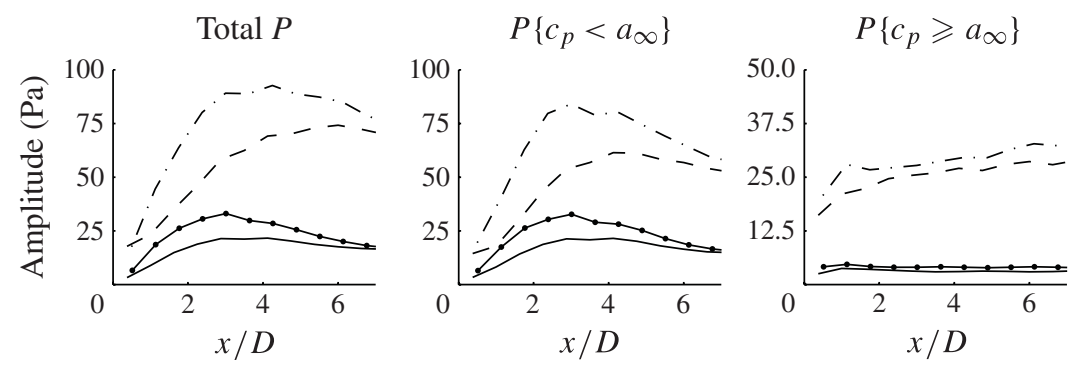

FIGURE 7. Fourier decomposition of pressure by phase speed for the cold $M_{\infty}=0.5$ jet (full curve), cold $M_{\infty}=0.9$ jet (dashed curve), hot $M_{\infty}=0.5$ jet (full curve with points) and hot $M_{\infty}=0.9$ (dash-dotted curve) jet at $m=0$. Note the different scaling of the ordinate for the supersonic component.

for short and long waves. The long-wave error is particularly important in the present context.

An alternative decomposition is based on two-point correlations of pairs of microphones. The POD (Lumley 1967) provides an optimal mechanism by which a set of measurements may be separated into uncorrelated components, ordered by energy, and so suits the problem at hand. To obtain smooth spectra, we divide the time 
series of instantaneous snapshots $P(x, r, \theta, t)$ into ensembles. We then transform each ensemble in both time and azimuthal angle, to obtain the set $\widetilde{P}_{m, \omega}^{k}(x, r)$, where $k$ is the bin number. For clarity of presentation we omit the subscript pair $(m, \omega)$ from all variables in what follows. The cross-spectral density tensor is defined as

$$
R_{i j}^{k}=\widetilde{P}_{i}^{k *} \cdot \widetilde{P}_{j}^{k}
$$

where $i$ and $j$ range from 1 through $N_{\text {ring }}$, and $\widetilde{P}_{j}^{k}$ is the transformed measurement in ensemble $k$, evaluated at $x=x_{j}$. We then form the ensemble-averaged cross-spectral density tensor,

$$
\boldsymbol{R}=\left\langle R^{k}\right\rangle=\frac{1}{N_{\text {ens }}} \sum_{k=1}^{N_{\text {ens }}} R^{k},
$$

and solve the eigenvalue problem

$$
\boldsymbol{R} \boldsymbol{x}=\lambda \boldsymbol{x} .
$$

Here $\boldsymbol{R}$ is Hermitian and positive definite by construction, so that $\lambda_{j} \geqslant 0$ and eigenvectors corresponding to different eigenvalues are orthonormal. The $\lambda_{j}$ are ordered such that $\lambda_{j+1} \leqslant \lambda_{j}$. A faster rate of decay of the $\lambda_{j}$ series indicates a higher correlation or coherence in the data. This is because the POD modes $x_{i}$ are uncorrelated, which follows from their orthonormality: $\left\langle x_{i}, x_{j}\right\rangle=\delta_{i j}$, where $\delta_{i j}$ is the Kronecker delta.

The cross-spectral density tensor $\boldsymbol{R}$ can be reconstructed from the POD modes:

$$
R_{i j}=\sum_{n=1}^{N_{\text {ring }}} \lambda_{n} x_{i}(n)^{*} x_{j}(n) .
$$

This decomposition allows the POD filtering of the measured data $\widetilde{P}$, where only the highest-energy mode is retained,

$$
\operatorname{POD}(\widetilde{P})=\sqrt{\lambda_{1}} x_{1}
$$

The application of the POD to the present data is discussed in the next section.

\subsection{Comparison with POD-filtered pressure}

In this section we present PSE predictions for all four jets in table 1 and compare them with both unfiltered and POD-filtered data.

Figures 8 and 9, respectively, show the evolution of pressure amplitude and phase along the microphone array for the cold $M_{\infty}=0.5$ jet. The PSE predictions capture the evolution of unfiltered amplitude well up to saturation, at least for the higher frequencies shown. After the peak, the two diverge and increasingly so at higher azimuthal modes and frequencies. This is presumably due to contamination of the measurements from uncorrelated acoustic fluctuations. This contamination is most readily apparent from the ensemble-averaged phase measurements in figure 9. The phase speed is inversely proportional to the slope of these curves. In some cases the slope suggests a very high phase-speed. It should be remembered that phase-speed is here measured along the microphone array. For example, an acoustic wave propagating normal to the array would register an infinite phase speed. This is, however, not consistent with the behaviour of a hydrodynamic wave having a convective phase speed and a direction of propagation roughly tangent to the array. 
(a)

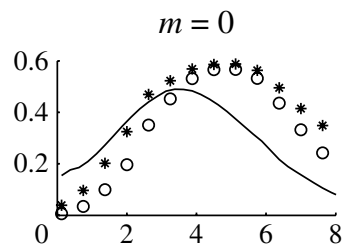

(b)

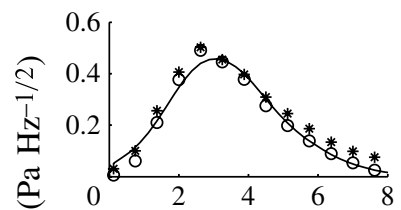

(c)

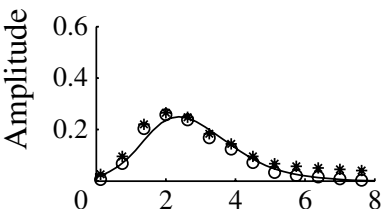

$(d)$

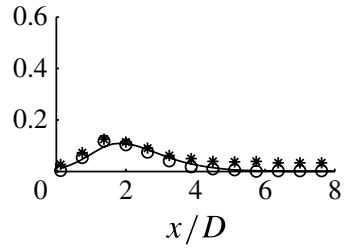

$m=1$
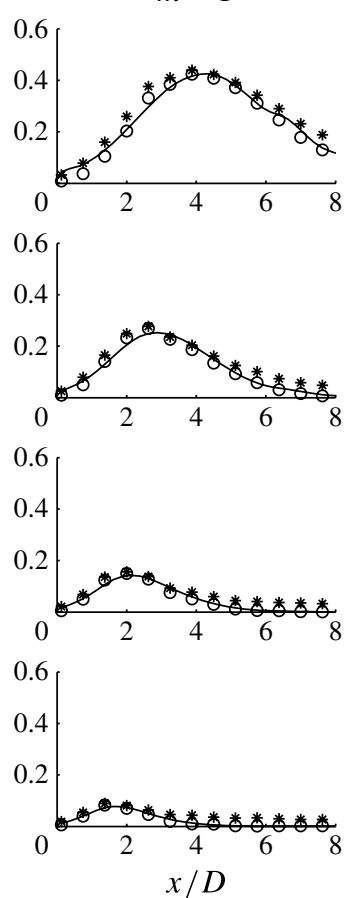

$m=2$
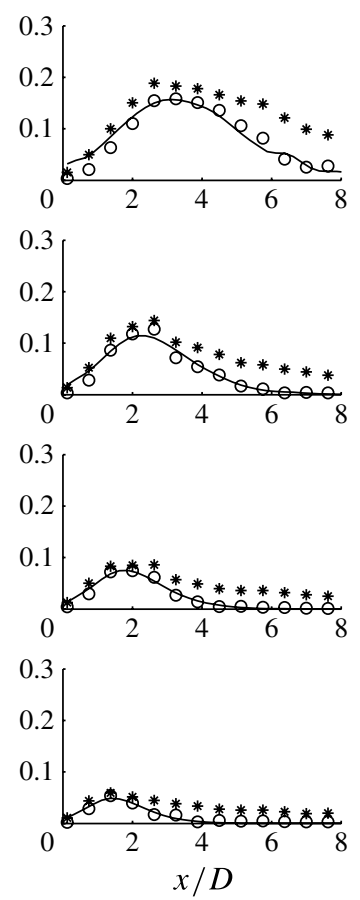

FIGURE 8. Pressure amplitude along the microphone array for the cold $M_{\infty}=0.5$ jet: r.m.s. data (*), first POD mode (o) and PSE predictions (-), at frequencies of $S t=0.20(a)$, $0.35(b), 0.5(c)$ and $0.65(d)$. Note $m$-dependence of ordinate.

We now turn our attention to the POD-filtered measurements. The effectiveness of the filtering is striking, particularly at the higher frequencies considered. Here, the POD-filtered phase closely follows that of the PSE prediction and similarly for the amplitude. This is the case even at frequencies as high as $S t=0.65$, considerably removed from that of the most amplified instability for this jet. (The agreement deteriorates at frequencies higher than $S t=0.65$ (not shown), but the streamwise spacing of the microphones becomes comparable to the wavelength of the instability wave at these higher frequencies and precludes drawing any conclusions from the present data regarding the applicability of linear stability theory.)

At the lowest frequency shown, particularly for the $m=0$ mode, the amplitude prediction is not as good, and this trend continues at even lower frequencies (not shown). For these cases, it appears that the growth rate is significantly underpredicted. This is probably due to stronger non-parallel effects, as the wavelengths for these modes become comparable with the potential core length. We suspect that the $m=0$ mode is more greatly affected than $m>0$ because of its non-vanishing behaviour (and scaling with the jet diameter at low frequencies) within the potential core, rendering it more sensitive to non-parallel effects at low frequencies. To counter this, the parabolizing assumption in (2.4) would need to be relaxed, resulting in a global method (Chomaz 2005), which we do not consider further.

We now consider the higher-Mach-number $\left(M_{\infty}=0.9\right)$ cold jet. Figure 10 shows the evolution of pressure amplitude and phase along the microphone array for $S t=0.35$; the full complement of frequencies with $0.2<S t<0.65$ is shown in the Appendix. 
(a)

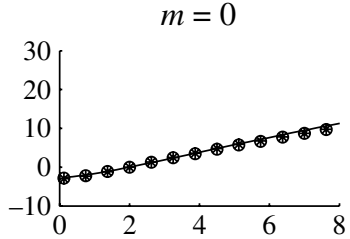

(b)

(c)

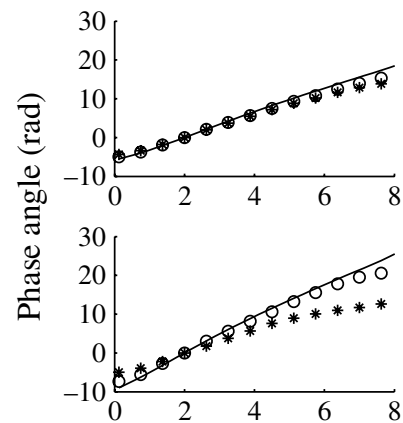

$(d)$

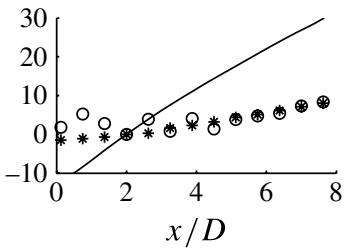

$m=1$
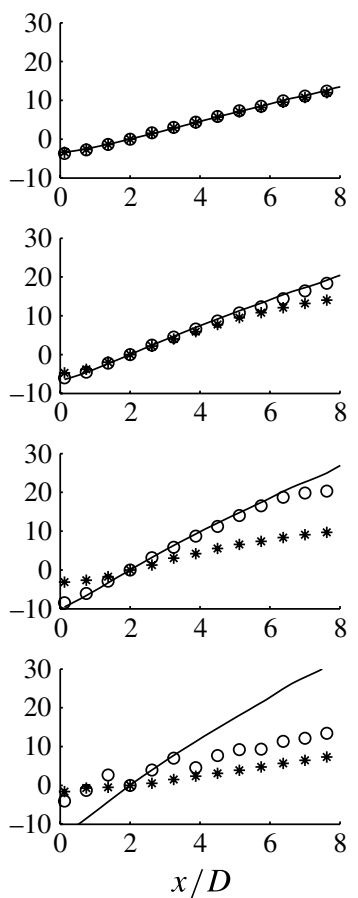

$m=2$
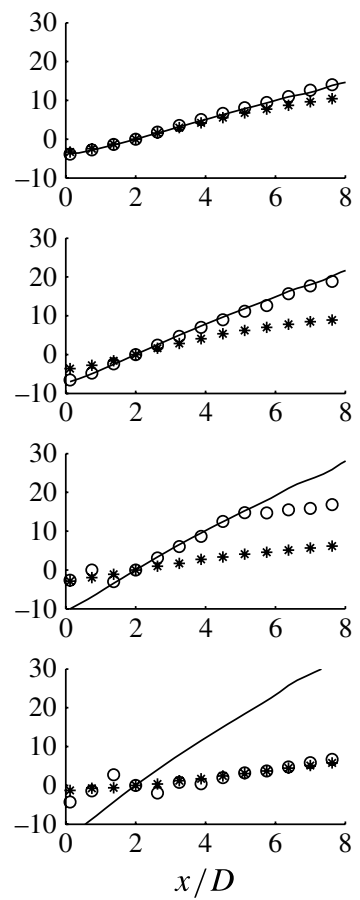

FIGURE 9. Phase along the microphone array for the cold $M_{\infty}=0.5$ jet: ensemble average $(*)$, first POD mode (o) and PSE predictions (-), at frequencies of $S t=0.20(a), 0.35(b)$, $0.5(c)$ and $0.65(d)$.

The data yield similar conclusions to the $M_{\infty}=0.5$ data, except at the highest frequency with $m>0$, where the PSE predictions correspond better with the second most energetic POD mode. As was the case for the cold $M_{\infty}=0.5$ jet, the benefits of POD filtering are considerable. The relative improvement is greater for the higherspeed jet, as it is apparently more contaminated (see discussion in $\S 4.1$ ) by acoustic waves at the downstream microphone positions. (This jet additionally suffers from an unidentified noise source that causes some ring-to-ring oscillations in the first half of the potential core. SC discuss this phenomenon and suggest that it is related to the internal aerodynamics of the nozzle. They show that the resonant peaks of the disturbance are consistent with those of duct-acoustic modes in quiescent space (see appendix B of their paper). It so happens that, for $m=0$, the first resonant peak occurs at $S t=0.35$, the same frequency as that presented in figure 10. Despite this, the agreement between the PSE and POD-filtered data is good.)

We now turn our attention to the two hot jets. Again, for brevity, we focus here on the behaviour at $S t=0.35$, with other frequencies leading to similar conclusions, as shown in the Appendix. It has been shown (Monkewitz \& Sohn 1988; Lesshafft \& Huerre 2007) that sufficiently heated round jets are susceptible to absolute instability. Based on their analysis, this is precluded for the hot jets in this work, due to both their relatively low temperature and their high speed. However, despite remaining in the convectively unstable regime, heating does have a destabilizing effect, particularly for the $m=0$ mode, as noted previously by SC. This is illustrated in figure 11, where we compare growth rates of the hot and cold $M_{\infty}=0.5$ jets. 

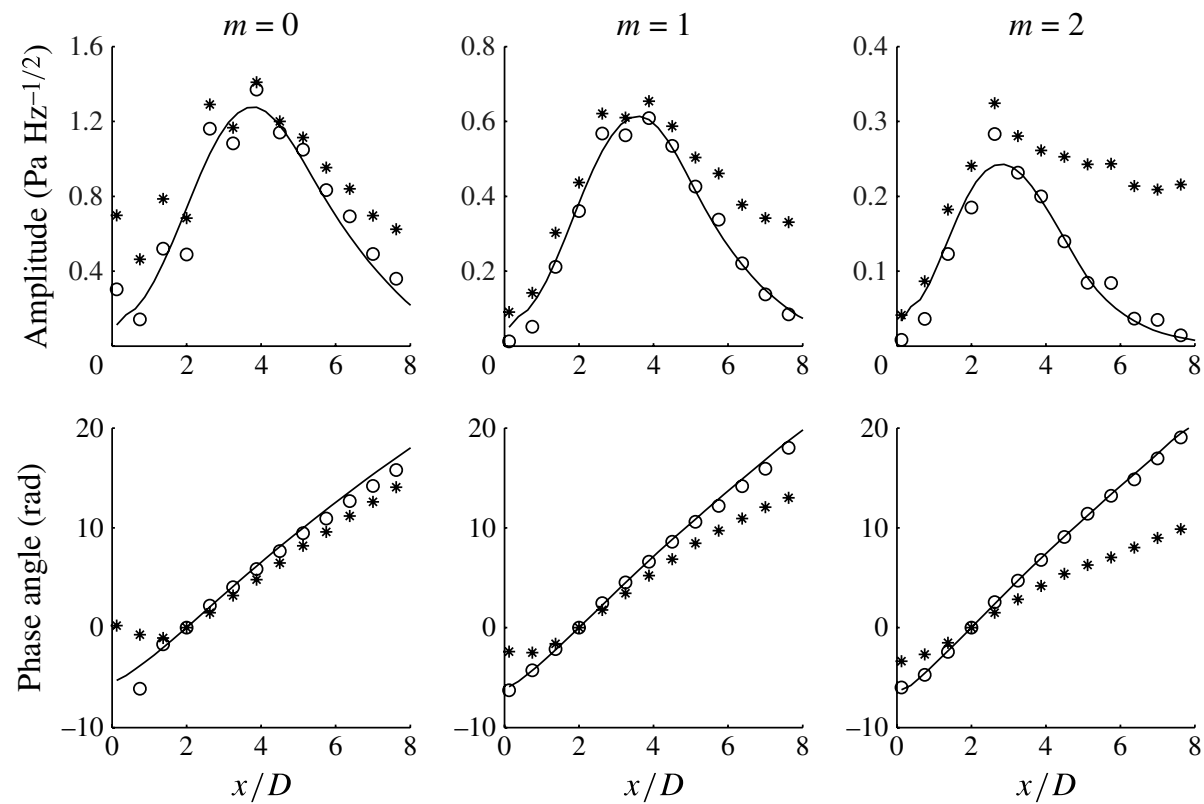

FIGURE 10. Pressure amplitude and phase along the microphone array for the cold $M_{\infty}=0.9$ jet: unfiltered measurements (*), first POD mode (o) and PSE (-), at a frequency of $S t=0.35$.
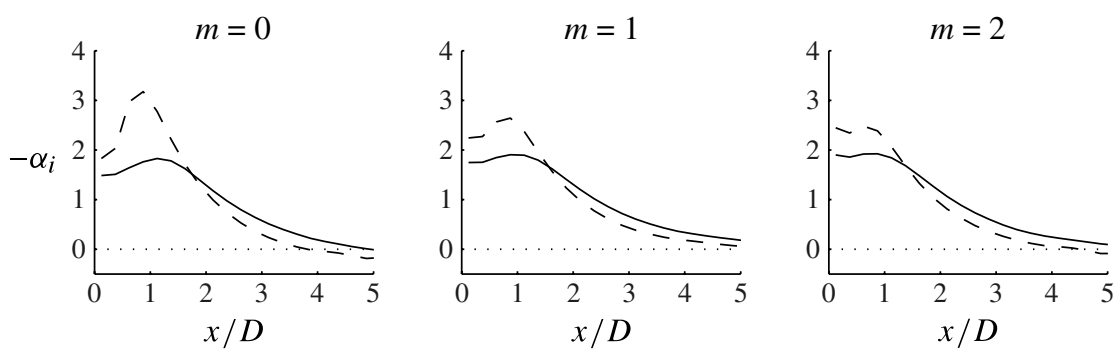

FIGURE 11. Growth rates $-\alpha_{i}$ for the cold (full curve) and hot (dashed) $M_{\infty}=0.5$ jets, at a frequency of $S t=0.35$.

The growth rate of the axisymmetric mode is nearly doubled. Figure 12 shows the evolution of pressure amplitude and phase along the microphone array for the hot $M_{\infty}=0.5$ jet at $S t=0.35$. The effects of destabilization and $m$ dependence thereof can be observed when we compare the amplitudes with those of the corresponding cold jet in figure $8(b)$. Here we must note that the amplitudes cannot be compared directly among the different operating conditions, as the microphone array was shifted upstream for the heated jets. This eliminated the first two microphone rings and displaced the remaining rings outwards by $\Delta r=0.25 D$, at which point the pressure signal has decayed further. The destabilization can then be appreciated by noting that the peak-amplitude ratios of the hot to cold jet (from figures 12 and $8(b)$ ) are approximately $1,0.7$ and 0.7 for $m=0-2$, respectively.

The match between the PSE prediction and the POD-filtered measurements is again very good, with some deterioration becoming evident for $m=2$ at the downstream 

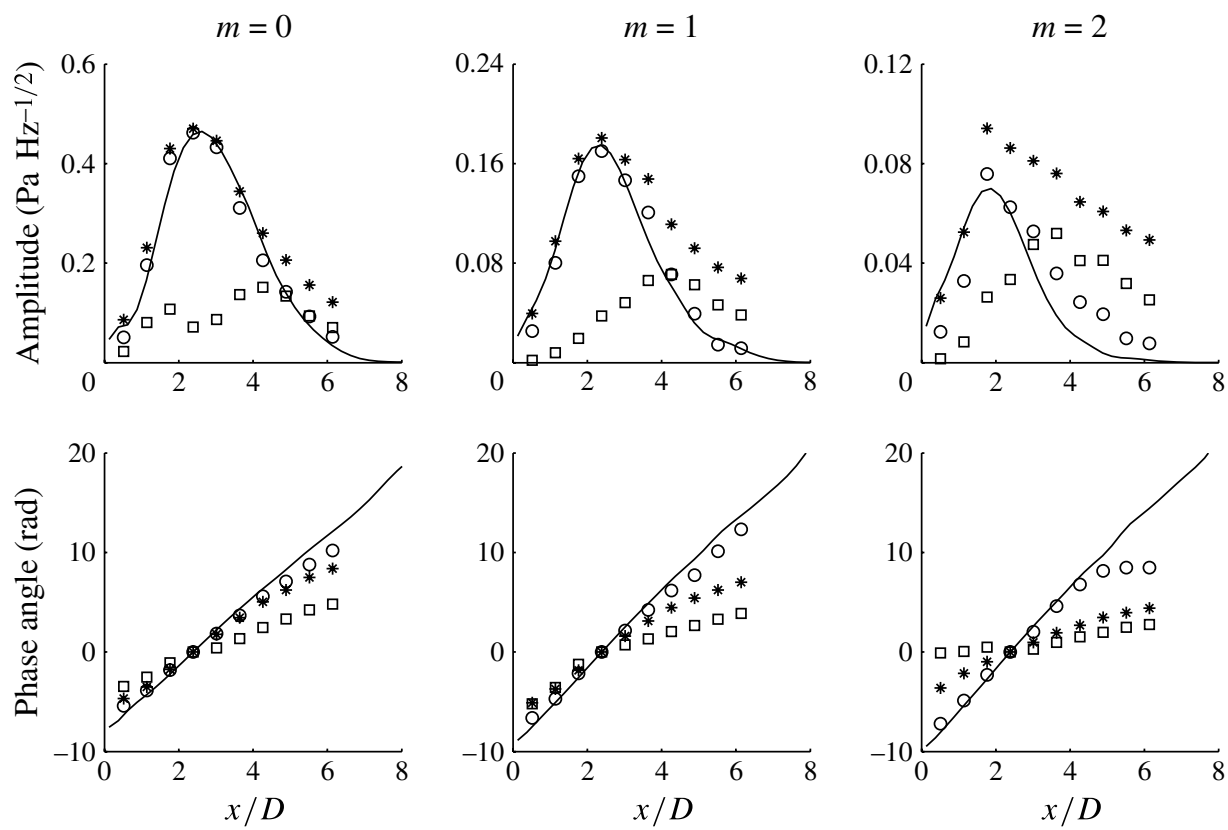

FIGURE 12. Pressure amplitude and phase along the microphone array for the hot $M_{\infty}=0.5$ jet: unfiltered measurements (*), first (o) and second $(\square)$ POD modes and PSE (-), at a frequency of $S t=0.35$. PSE amplitudes are based on first POD mode.

microphone positions. There is apparently mixed hydrodynamic-acoustic behaviour of the POD-filtered pressure in this instance. Figure 13 shows analogous data for the hot $M_{\infty}=0.9$ jet. Similar observations can be made here, but are blurred by the greater degree of acoustic contamination. In particular, at $m=2$, the PSE prediction matches better with the second POD mode, as for the cold $M_{\infty}=0.9$ jet in figure 10 . This is further exaggerated at higher frequencies, where the PSE prediction fits better with the second POD mode for all azimuthal modes considered (see figures 22-25 in the Appendix).

Up to the undetermined constant (at each frequency and azimuthal wavenumber) associated with any linear theory, the results in this section show convincing agreement between the PSE predictions and the phase and amplitude envelope of the POD-filtered pressure measured along the microphone array. For the present measurements, the conclusion applies up to frequencies of about $S t=0.65$, after which the microphone spacings are too large to provide an unambiguous assessment. At frequencies below about $S t=0.2$, and particularly for the axisymmetric mode, there are increasing discrepancies that appear to be related to non-parallel effects that render the PSE approximation progressively less valid. These discrepancies do not rule out an instability wave theory for these frequencies, but it may be necessary to use global modes to achieve quantitative agreement.

The POD filtering is largely effective at isolating the instability wave as the most energetic POD mode of the experimental data, demonstrating that the evanescent pressure fluctuations associated with instability waves comprise the dominant contribution to the measurements. For certain conditions, particularly higher frequencies and azimuthal modes, the second most energetic POD mode agrees well with the PSE predictions, while the most energetic mode has a phase speed 

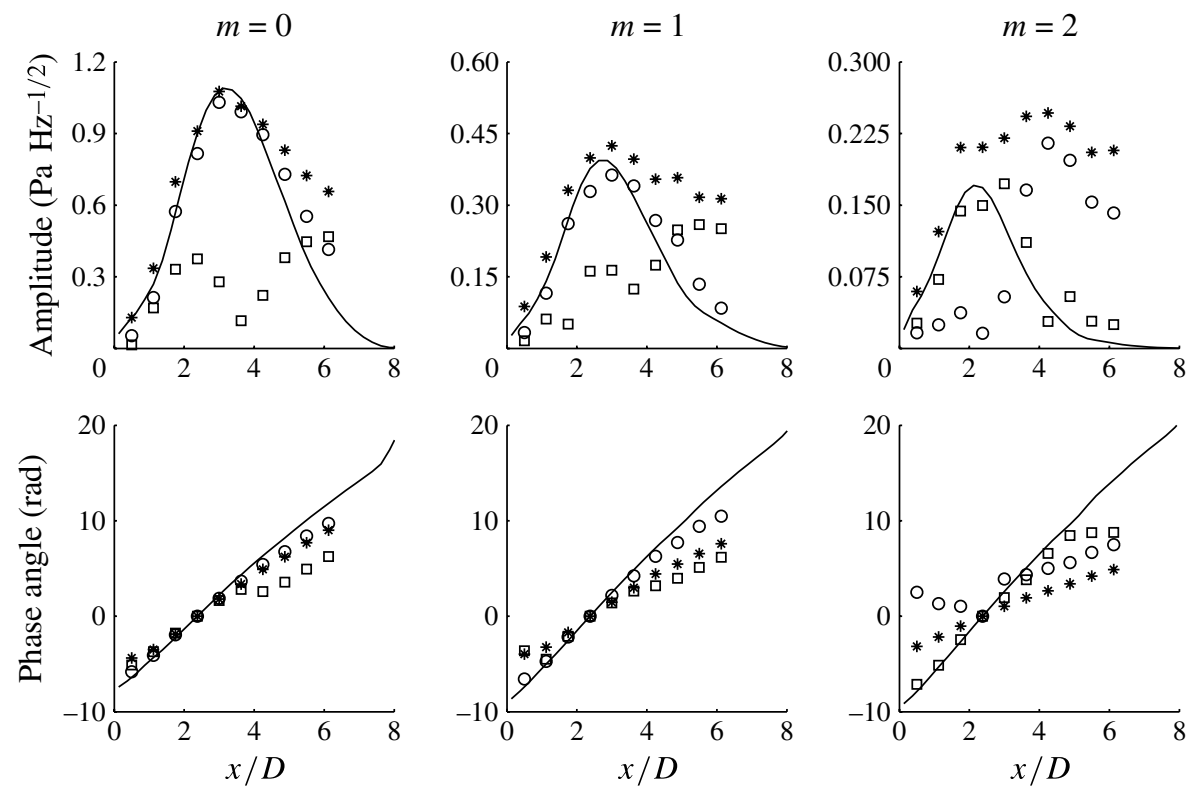

FIGURE 13. Pressure amplitude and phase along the microphone array for the hot $M_{\infty}=0.9$ jet: unfiltered measurements $(*)$, first $(\circ)$ and second $(\square)$ POD modes and PSE $(-)$, at a frequency of $S t=0.35$. PSE amplitudes are based on first POD mode, except for at $m=2$, where the second POD mode is used.

consistent with an acoustic contribution to the microphone pressure that is apparently uncorrelated with the instability wave.

\section{Detecting instability waves in measured velocity fluctuations}

While the previous section provides strong evidence for the efficacy of linear PSE in describing the large-scale structures in turbulent jets, it seems worthwhile to check for consistency of the inferred amplitude of instability waves with near-field velocity data. Such agreement, if found, would constitute stronger evidence for the linear theory, but it is not clear a priori to what extent such structures can be cleanly detected in measured near-field velocity fluctuations that comprise a far richer spectrum of turbulence scales. This is because the fluctuating pressure field of a turbulent jet can be considered to be a convolution of the velocity field (cf. the pressure Poisson equation in incompressible flow), and, as such, it has more rapid spectral decay, in both temporal frequency and azimuthal wavenumber. In the inertial subrange of incompressible flow, for example, the pressure spectrum decays with an exponent of $-7 / 3$ (George, Beuther \& Arndt 1984), compared with the well-known $-5 / 3$ exponent of the velocity spectra.

To examine this question, we apply the POD to a series of cross-sectional PIV snapshots of the cold $M_{\infty}=0.9, R e=16 \times 10^{5}$ jet in table 1 . The snapshots, taken $\Delta t=0.1 \mathrm{~s}$ apart, can be considered uncorrelated, as $U_{\text {jet }} \Delta t \approx 600 D$ for this jet. We briefly describe the construction of the POD modes of velocity; consult Gudmundsson (2010) for further details. We start with $N$ instantaneous snapshots of streamwise velocity $U_{m}(r, t)$, where $m$ denotes the azimuthal mode number. The POD modes $\phi_{j}(r)$ 
are formed via linear combinations of the $U_{m}(r, t)$,

$$
\phi_{j}(r)=\sum_{k=1}^{N} c_{k}^{j} U_{m}\left(r, t_{k}\right)
$$

The $c_{j}$ are the eigenvectors of the covariance matrix $\boldsymbol{M}$, where

$$
\begin{aligned}
M_{k l} & =\left\langle U_{m}\left(r, t_{k}\right), U_{m}\left(r, t_{l}\right)\right\rangle \\
& =\int_{0}^{\infty} U_{m}\left(r, t_{k}\right) U_{m}^{*}\left(r, t_{l}\right) r \mathrm{~d} r .
\end{aligned}
$$

Eigenvalue $\Lambda_{j}=\left\|\phi_{j}\right\|^{2}$ of $\boldsymbol{M}$ represents the energy of POD mode $j$, where the $\Lambda_{j}$ are ordered such that $\Lambda_{j} \geqslant \Lambda_{j+1}$. Then, for example, $\phi_{1}$ is the linear combination of the $U_{m}$ that has the highest energy of all such combinations satisfying $c^{j \mathrm{~T}} \cdot c^{j}=1$.

Optimally, one would use time-resolved velocity measurements to construct the POD modes. This would allow a frequency-dependent comparison, presumably optimized at the energy-dominant frequency. The present velocity data are not time-resolved, however. We therefore consider two alternative procedures for comparing the data with PSE. In the first case, we compare both the mean-squared (unfiltered) PIV data, and the POD modes of the PIV data, to the frequency-averaged PSE solution. As different frequencies need not be correlated, this tacitly assumes that the behaviour is dominated by a globally dominant frequency for each azimuthal wavenumber. In the second case, we explicitly search for this dominant frequency by looking for a correlation between the radial structure of each PSE eigenfunction and each POD mode.

Turning to the frequency-averaged approach, the instability wave of azimuthal mode $m$ is given by

$$
u_{m}(x, r, t)=\sum_{n=1}^{N_{i}} A_{m}(n \omega) \hat{u}_{m}(x, r, n \omega) \mathrm{e}^{\mathrm{i} \int^{x} \alpha_{m}(\xi, n \omega) \mathrm{d} \xi} \mathrm{e}^{-\mathrm{i} n \omega t},
$$

where $A_{m}(n \omega)$, defined in (3.7), is the amplitude of the instability wave as determined using the POD-filtered microphone data, $\hat{u}_{m}(x, r, \omega)$ is the normalized eigenfunction, and $N_{i}$ is the number of frequencies retained. The mean-squared instability wave (i.e. the mean square of (5.3)) is given by

$$
\frac{1}{N} \sum_{k=1}^{N}\left|u_{m}\left(x, r, t_{k}\right)\right|^{2}=\sum_{n=1}^{N_{i}} A_{m}(n \omega)^{2}\left|\hat{u}_{m}(x, r, n \omega)\right|^{2} \mathrm{e}^{-2 \int^{x} \alpha_{i m}(\xi, n \omega) \mathrm{d} \xi},
$$

where $\alpha_{i m}=\operatorname{Im}\left[\alpha_{m}\right]$.

Figure 14 shows the mean-squared instability wave, the unfiltered PIV data and the first POD mode. As expected, the instability wave corresponds to a relatively small portion of the overall fluctuation energy (compared to that observed in the pressure fluctuations). The radial distributions (not surprisingly) do not correspond particularly well with the total (unfiltered) data, although some general features, such as the radial location of maxima, are captured.

Before discussing the agreement with POD-filtered velocity fields, we note several issues related to the data that are evident in figure 14. First, there are instances (e.g. figure $14(c), m=2$ ) where the energy of the instability wave exceeds that in the data. This cannot be the case, by definition, as the data contain fluctuations from all sources, not just instability waves. The reason for the overshoot is an overestimation of the instability wave amplitude $A_{m}(\omega)$ at low frequencies. The amplitude decays 

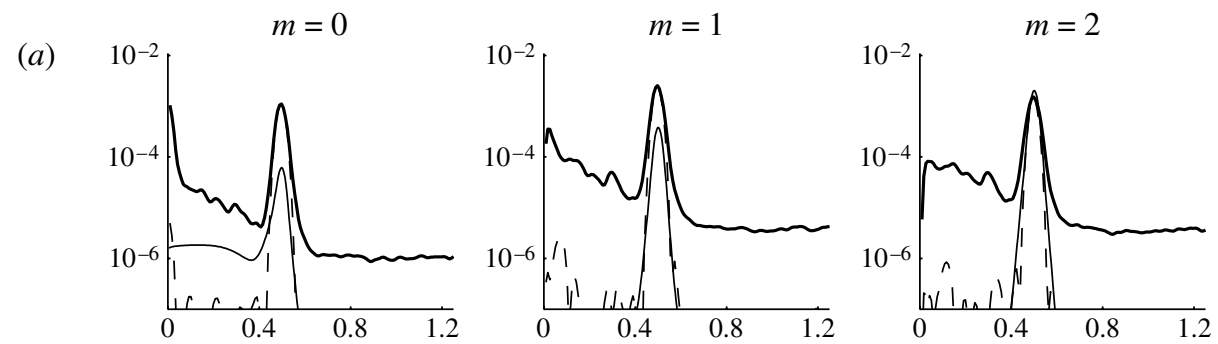

(b)
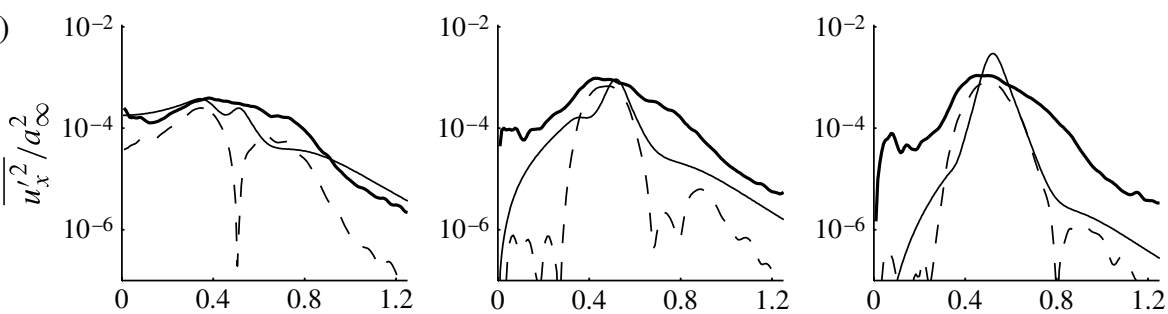

(c)
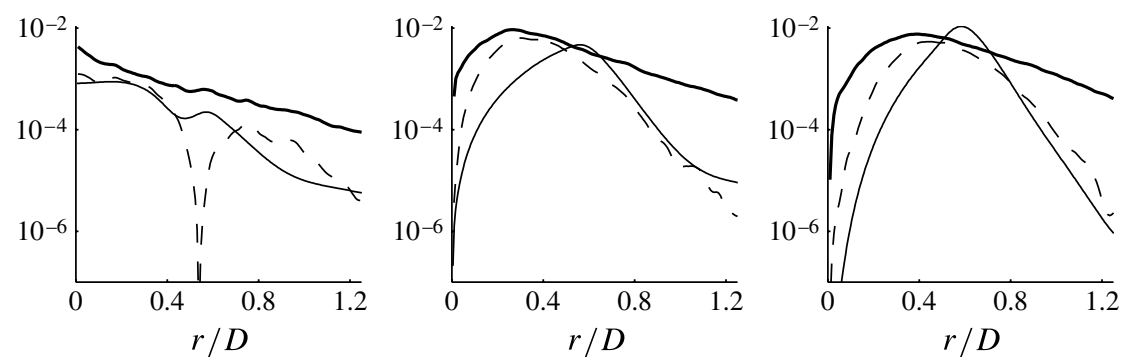

FIGURE 14. Mean-squared amplitude of the PIV measurements (thick full curve), scaled instability waves (thin full curve) and first POD mode (dashed curve) of the cold $M_{\infty}=0.9$ jet, at $x / D=0.5(a), 3.5(b)$ and $8(c)$.

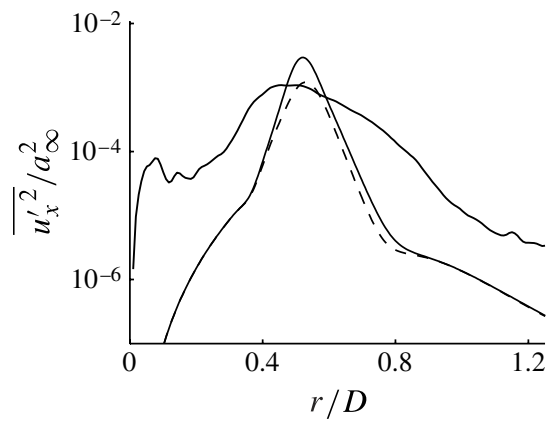

FIGURE 15. Mean-squared amplitude of the PIV measurements (thick full curve), scaled instability waves (thin full curve) and scaled instability wave with $S t=0.05$ omitted from (5.4) (dashed curve). Azimuthal mode $m=2$; cross-section $x / D=3.5$.

rapidly with increasing frequency so that the lowest frequencies contribute heavily in the mean-square calculation of (5.4). This is illustrated in figure 15, which shows case $(b) m=2$ of figure 14, but with the lowest frequency $(S t=0.05$ ) omitted from the sum in (5.4). Meanwhile, the instability wave representation of the PSE 

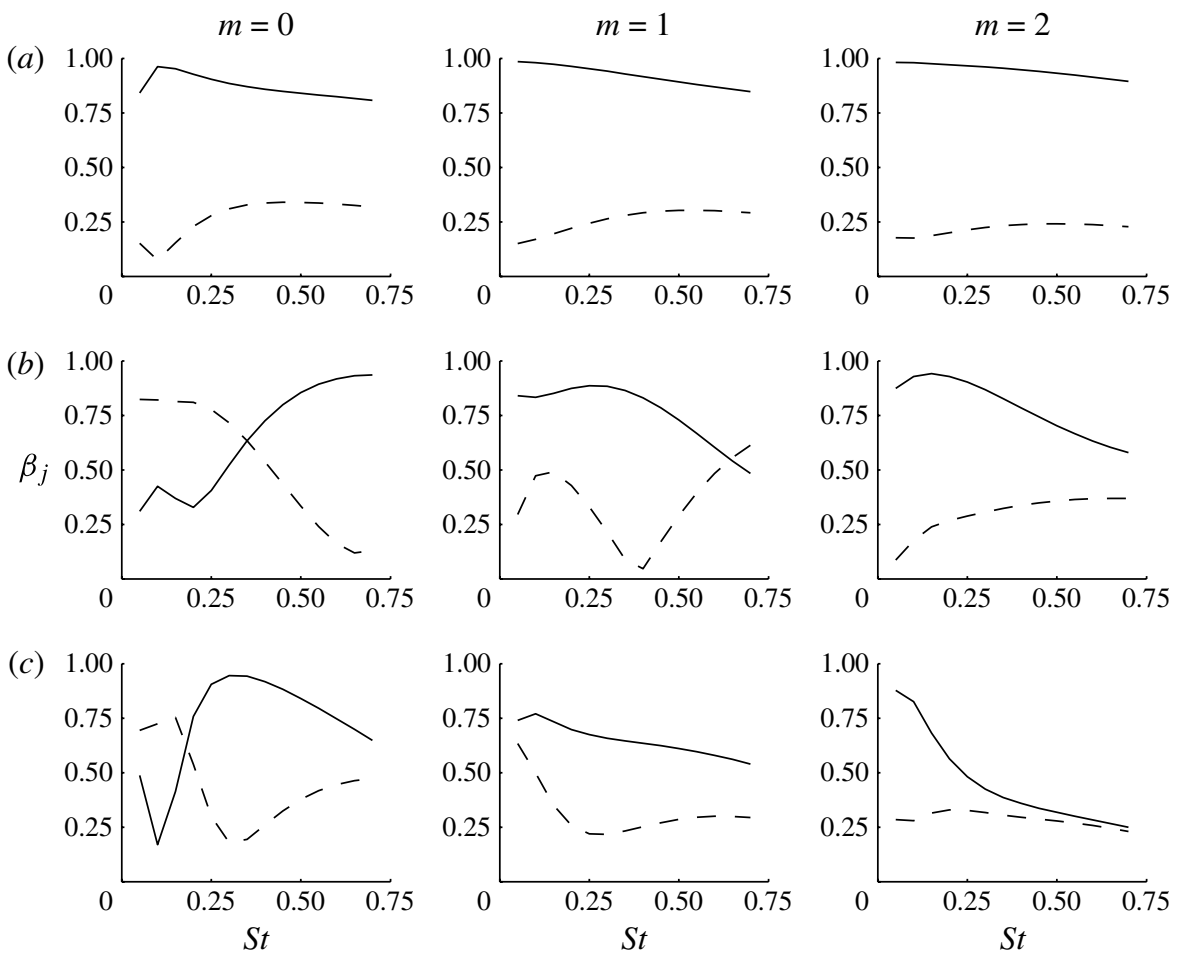

FIGURE 16. Correlation coefficient $\beta_{j}$, defined in (5.5): $j=1$ (full curve) and $j=2$ (dashed curve) at $x / D=0.5(a), 3.5(b)$ and $8(c)$.
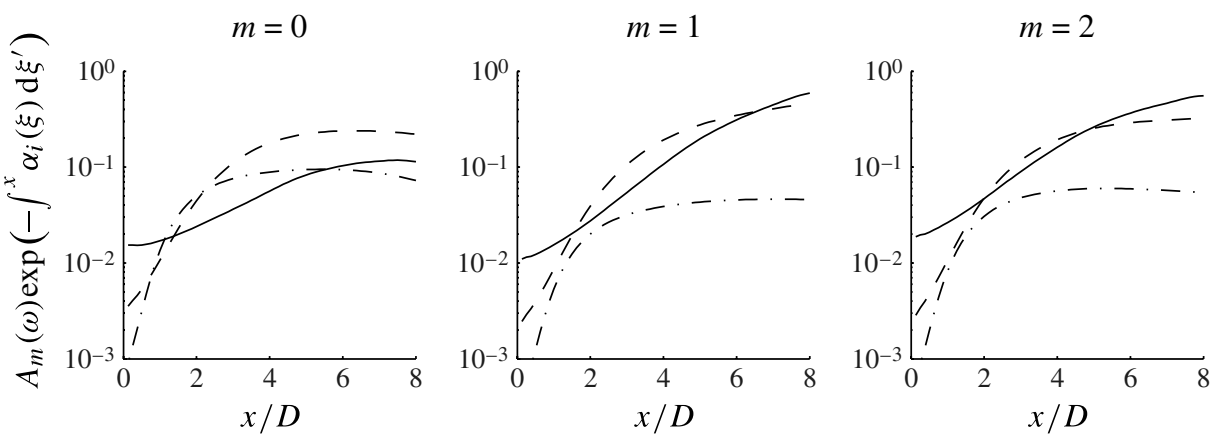

FIGURE 17. Scaled instability wave amplitudes at $S t=0.15$ (full curve), 0.35 (dashed curve) and 0.70 (dash-dotted curve).

is increasingly in error at low frequencies, as was discussed in $\S 4.2$. This renders an absolute comparison using estimated amplitudes difficult.

Moreover, we note that the agreement is poorer near the centreline. This is due to fluctuations unrelated to instability waves. This is particularly true near the nozzle lip, where fluctuations having significant energy appear intermittently in the PIV snapshots, strongly affecting the mean square. These fluctuations are likely to have the same source as the resonance phenomena discussed in appendix B of SC, where significant resonance peaks appeared in the near-nozzle pressure spectra. This resonance does not 
(a)

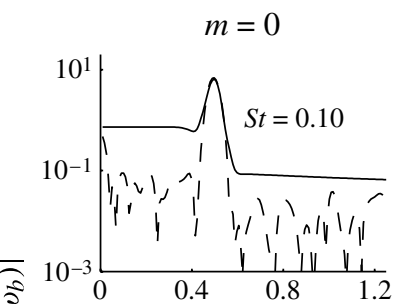

(b)

(c)

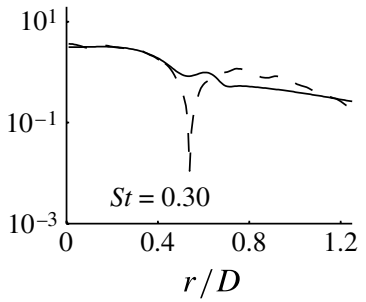

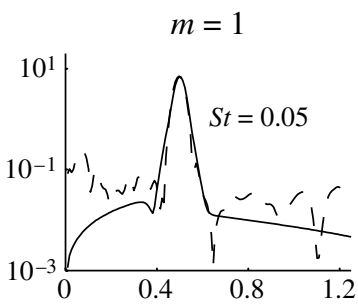
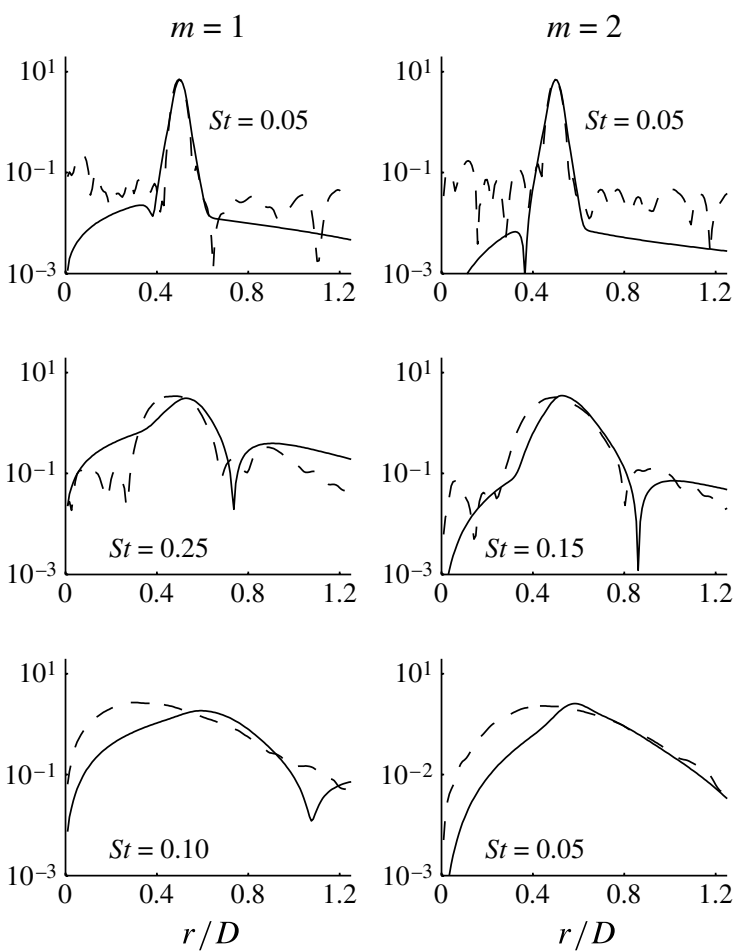

FIGURE 18. Radial distribution of POD mode $\phi_{1}$ (dashed curve) and scaled eigenfunctions $\beta_{1}\left(\omega_{b}\right) \hat{u}\left(x, r, \omega_{b}\right)$ (full curve), where $\omega_{b}$ is the frequency at which $\beta_{1}$ is maximized (shown in terms of the corresponding Strouhal number), at $x / D=0.5(a), 3.5(b)$ and $8(c)$.

appear in pressure spectra of the cold $M_{\infty}=0.5$ jet, nor in that of the heated jets. Bridges \& Wernet (2003) show centreline r.m.s. of both the PIV measurements of the cold $M_{\infty}=0.9$ jet discussed here, and also an $M_{\infty}=0.9$ jet with $T_{j e t} / T_{\infty}=2.7$; elevated near-nozzle fluctuations are only seen for the colder jet. Further, the degree of near-nozzle centreline velocity fluctuations seen for the present jet does not appear to have been reported in other datasets, for example those of Crow \& Champagne (1971), or Zaman \& Hussain (1980). Note, however, that the POD filtering effectively removes these fluctuations, underlining that they are not correlated with instability waves. Outside the shear layer there is also a greater discrepancy, which could at least in part be ascribed to uncertainty in the PIV data, whose quality decays rapidly outside the jet, as the light-reflecting particles become scarce.

We now turn to the comparison between the first POD mode of the velocity fluctuations and the frequency-averaged PSE results, also shown in figure 14. A priori, we do not naturally expect a favourable match between the two, as the frequency averaging adds together instability modes that are not necessarily correlated, while the POD modes are uncorrelated with each other. Especially considering this and other issues discussed above, we observe an encouraging match in amplitude with the PSE predictions; the radial distribution of velocity shows excellent agreement close to the nozzle lip. Further downstream, the general trends continue to be captured, but, as might be expected based on the broader range of scales in the data at these locations, the quantitative agreement deteriorates. 

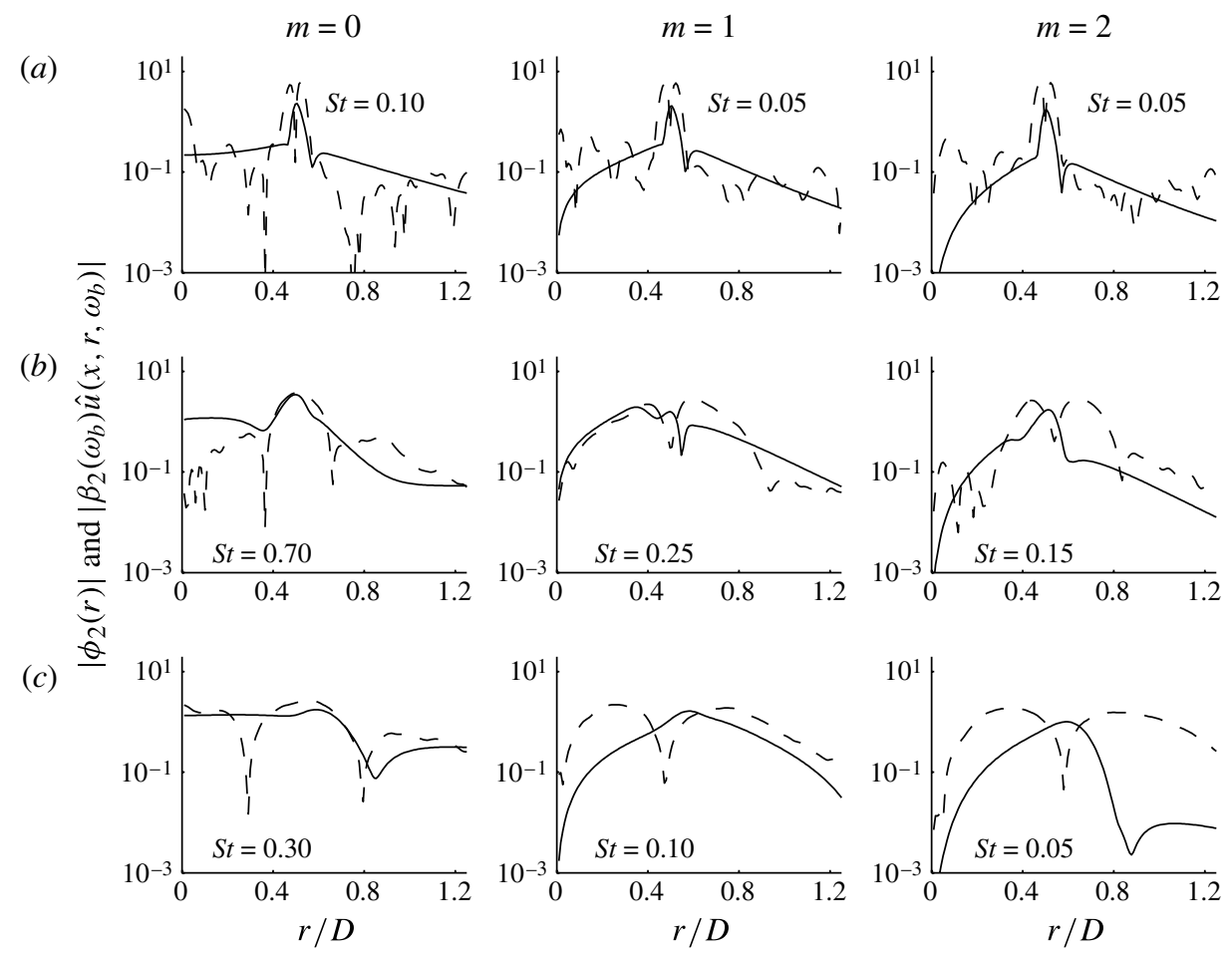

FIGURE 19. Radial distribution of POD mode $\phi_{2}$ (dashed curve) and scaled eigenfunctions $\beta_{2}\left(\omega_{b}\right) \hat{u}\left(x, r, \omega_{b}\right)$ (full curve); notation is the same as in figure 18 .

An alternative to integrating over all frequencies is to compare the POD modes to instability waves as a function of the frequency of the latter. The frequency at which the best match is obtained should then be close to the centre of the dominant frequency band, if one exists. To quantify this fit, we first renormalize the eigenfunctions $\hat{u}(x, r, \omega)$ and POD modes $\phi_{j}(x, r)$ such that $\|\hat{u}\|=\left\|\phi_{j}\right\|=1$. We then project the eigenfunctions onto the POD modes and record their correlation:

$$
\beta_{j}(\omega)=\left\langle\hat{u}(x, r, \omega), \phi_{j}(r)\right\rangle
$$

where the inner product is defined in (5.2). From the normalization of $\hat{u}$ and $\phi_{j}$ we see that $0 \leqslant\left|\beta_{j}\right| \leqslant 1$. This quantity is shown in figure 16 , where we include the first two POD modes $\left(\phi_{1}\right.$ and $\left.\phi_{2}\right)$. A higher correlation is in general obtained for $\phi_{1}$, as expected. However, there are cases, such as for $m=0$ at $x / D=3.5$, where there is a cross-over, with $\phi_{1}$ being well correlated at higher frequencies but not at lower frequencies, where $\phi_{2}$ in turn is well correlated. This illustrates how the low-order POD modes can all be associated with instability waves but at different frequencies.

Note also that low frequencies dominate near the nozzle $(x / D=0.5)$. At $x / D=3.5$ the best fit is obtained at higher frequencies, while at $x / D=8$ the best fit is again at the lower frequencies, with the exception of $m=0$. This might seem paradoxical, given that the most unstable frequency is a decreasing function of the streamwise distance. Here, however, we must take into account the local amplitude of each frequency. While being relatively stable, low-frequency waves have the highest amplitude near the nozzle. Higher-frequency waves grow in amplitude thereafter via their instability. Going further downstream these waves become stable and 
(a)

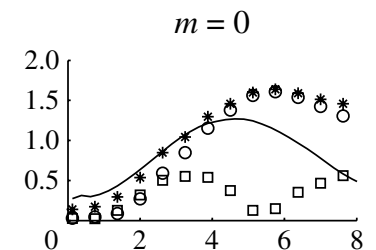

(b)

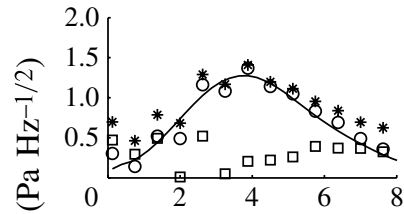

(c)

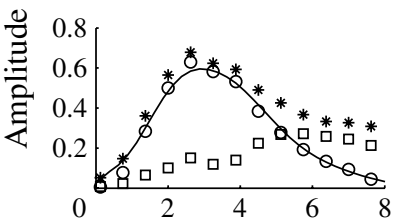

$(d)$

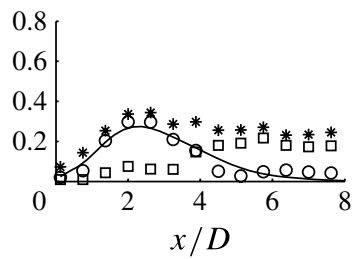

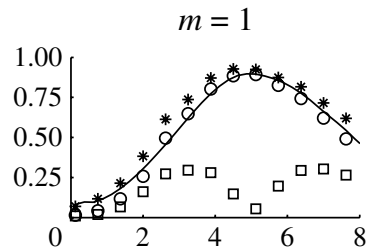
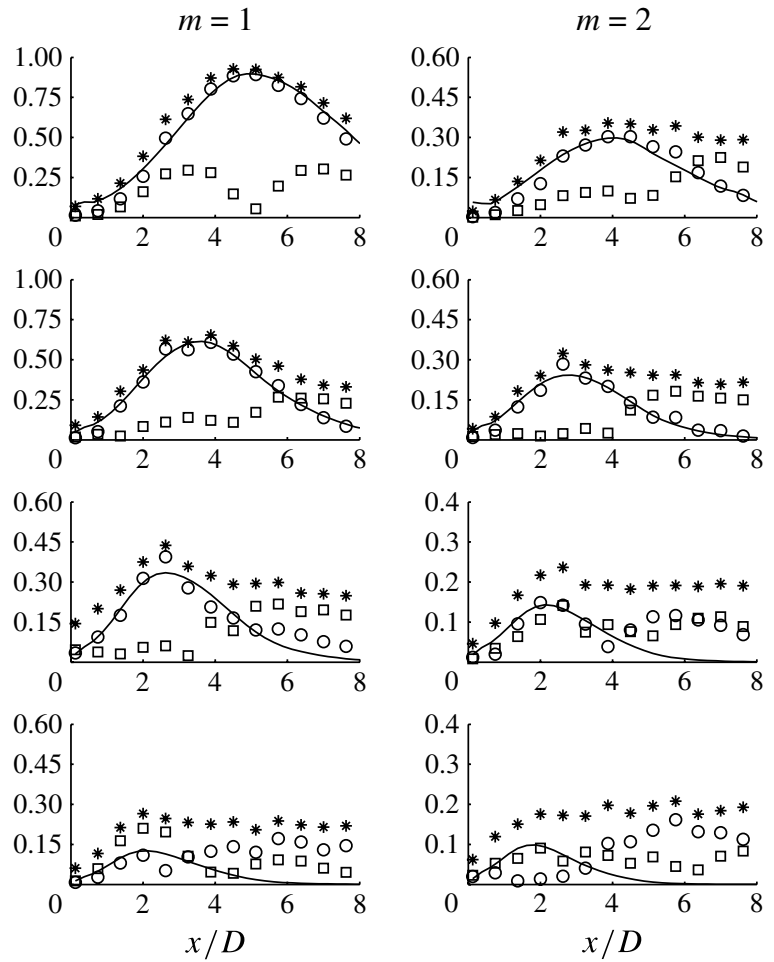
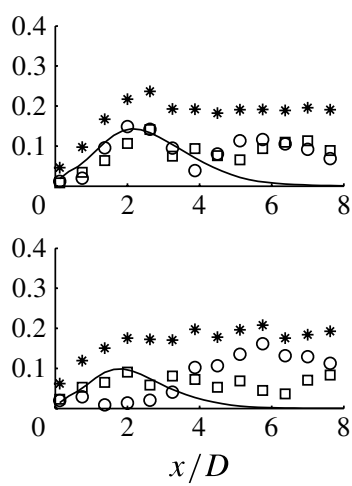

FIgURE 20. Pressure amplitude along the microphone array for the cold $M_{\infty}=0.9$ jet: r.m.s. data (*), first (o) and second ( $\square$ ) POD modes and PSE predictions (-), at frequencies of $S t=0.20(a), 0.35(b), 0.5(c)$ and $0.65(d)$. PSE amplitudes are based on first POD mode, except for $S t=0.65$ at $m=2$, where the second POD mode is used.

start decaying and do so sooner the higher their frequency. Meanwhile, the lowest frequencies are still growing and hence the best fit is again found at lower frequencies at $x / D=8$ (again excepting $m=0$ ). This development is illustrated in figure 17, showing the evolution of amplitude as calculated from PSE and scaled with $A_{m}(\omega)$, as determined using the microphone array. The dominant frequencies at $x / D=0.5,3.5$ and 8 in this figure correspond well with the best-fit trends at the same locations in figure 16, including the behaviour of $m=0$ at $x / D=8$.

We now look at the radial distribution of the POD modes $\phi_{j}(x, r)$ and the scaled eigenfunctions $\beta_{j}\left(\omega_{b}\right) \hat{u}\left(x, r, \omega_{b}\right)$, where $\omega_{b}$ is the frequency at which $\beta_{j}$ is maximized. These are shown in figures 18 and 19 for the first and second POD modes, respectively. With a few exceptions, the comparisons are very convincing. The exceptions all correspond to frequencies where the correlation, $\beta_{j}$, is smaller than about 0.5 .

To summarize, an analysis of the near-field velocity fluctuations from PIV confirms the presence of instability waves as predicted by the PSE and with an overall amplitude that is consistent with the values inferred by matching the pressure fluctuations along the microphone array. Overall, the agreement is not as sharp as it is with the microphone array, but it has been shown that this is probably due to limitations associated with using PIV snapshots that are not time-resolved. When we search for the dominant frequency by finding that frequency of the PSE solution 
$m=0$

(a)

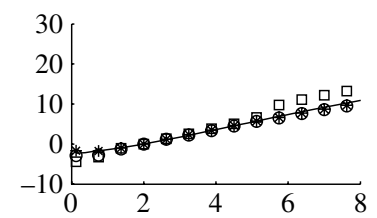

(b)

(c)
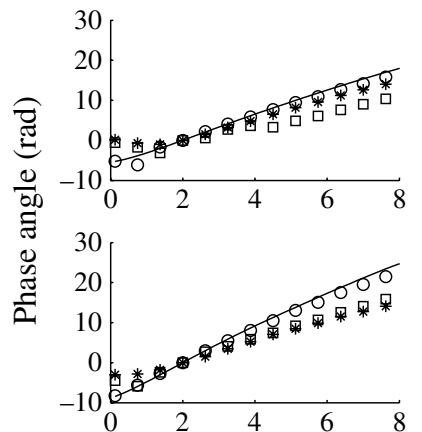

(d)

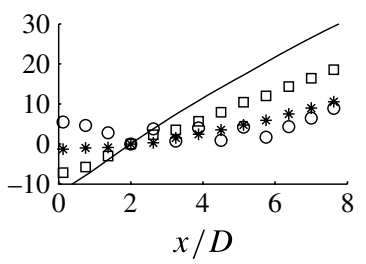

$m=1$
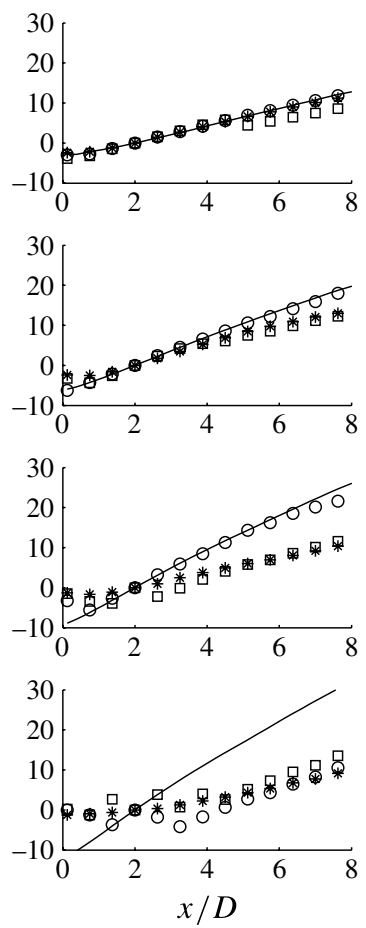

$m=2$
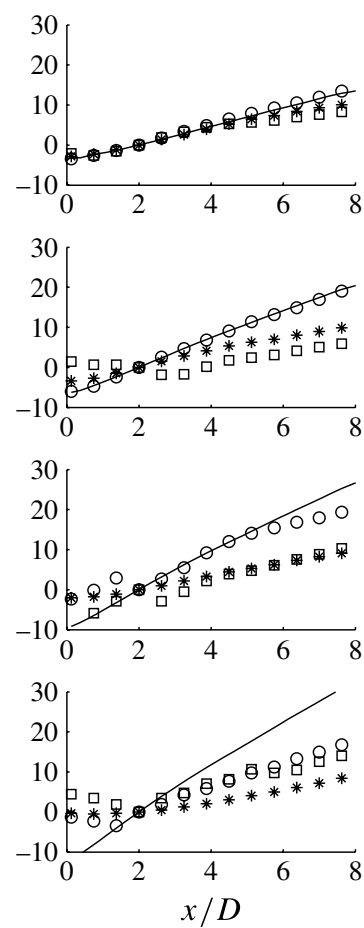

FIGURE 21. Phase along the microphone array for the cold $M_{\infty}=0.9$ jet: ensemble average $(*)$, first (o) and second ( $\square$ ) POD modes and PSE predictions (-), at frequencies of $S t=0.20(a), 0.35(b), 0.5(c)$ and $0.65(d)$.

which gives the highest correlation with the POD mode, there is substantially better agreement in shape and amplitude.

\section{Summary and conclusions}

In this work we have pursued modelling of large-scale coherent structures in natural (unforced), turbulent jets at high Reynolds number as linear disturbances to the turbulent mean flow field. We used linear PSE to predict the evolution of each frequency and azimuthal mode number, and compared the results to data from a caged microphone array placed just outside the jet shear layer, and to PIV snapshots of the near-field velocity at a number of jet cross-sections. In both cases, the data need to be treated carefully, as the expectation is that the contribution to the signal from the largescale structures represents only a portion of the signal. Apart from an indeterminate constant at each frequency and azimuthal mode, inherent to any linear theory, we find in each case that performing a POD filtering of the data, typically retaining only the most energetic or second most energetic mode, results in good agreement with the PSE predictions. Moreover, amplitudes inferred by choosing the arbitrary constant to match the microphone array are in reasonable agreement with near-field amplitudes implied by the PIV data.

The best agreement between the experimental data and the predictions occurs over the frequency range $0.2<S t<0.65$. The upper bound is determined solely by resolution limitations associated with microphone spacing along the array; we believe 
(a)

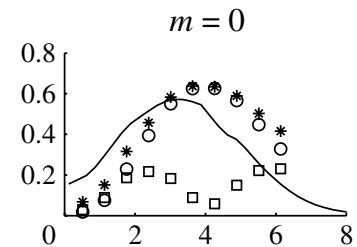

(b)

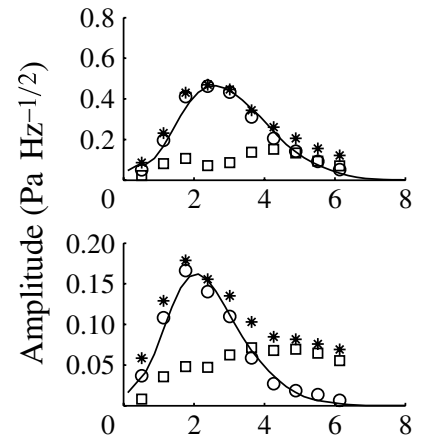

$(d)$

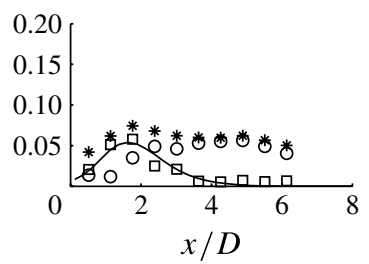

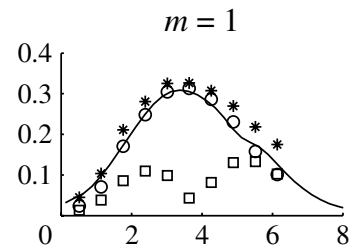
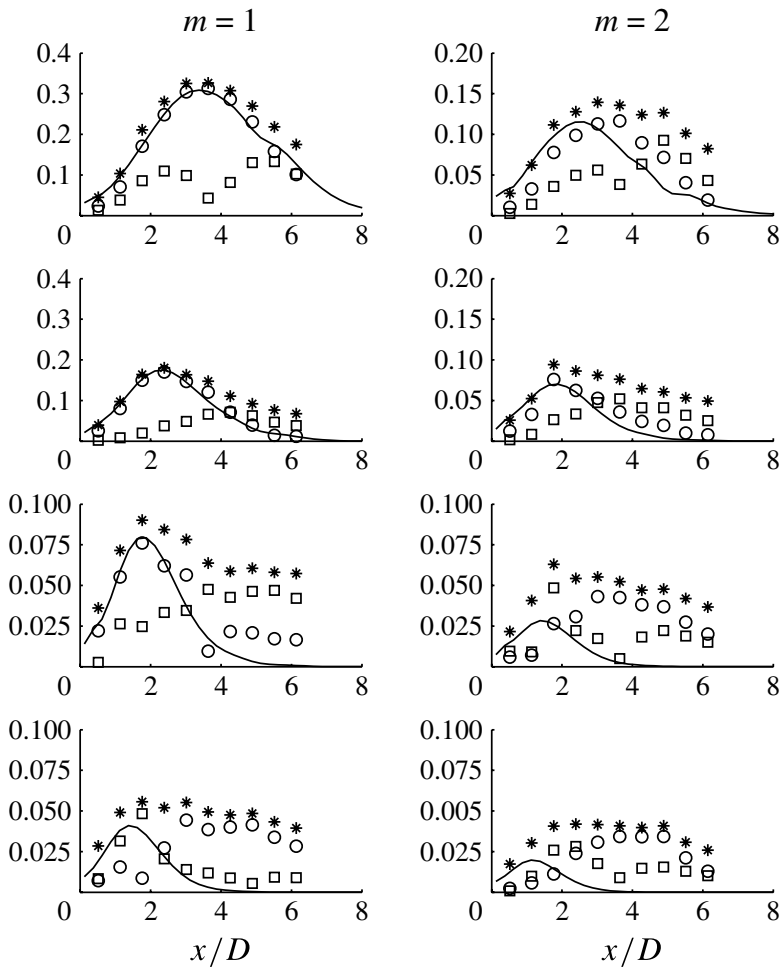
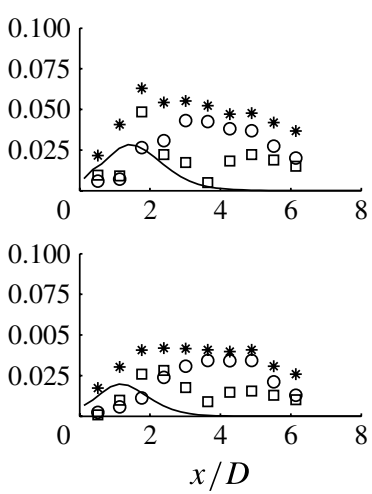

FIGURE 22. Pressure amplitude along the microphone array for the hot $M_{\infty}=0.5$ jet: r.m.s. data (*), first (o) and second ( $\square$ ) POD modes and PSE predictions (-), at frequencies of $S t=0.20(a), 0.35(b), 0.5(c)$ and $0.65(d)$. PSE amplitudes are based on first POD mode, except for all $m$ of $S t=0.65$, where the second POD mode is used.

that reasonable agreement could be obtained at higher frequencies with a denser array, although it will probably be more difficult to uniquely assess the instability wave contribution to the data at these frequencies, since they are only unstable very near the nozzle. At Strouhal numbers lower than 0.2, agreement deteriorates, which we believe is related to non-parallel effects that are not captured by PSE. A global mode analysis, while computationally challenging, may provide a more appropriate prediction for those frequencies.

The main conclusion that can be drawn from the comparisons is that, to reasonable accuracy, the average evolution of large-scale structures in natural, turbulent jets may be predicted based on linearized disturbances to the turbulent mean flow field. This conclusion is rather different from the conclusions of past studies that have focused on transitional jets, and on forced transitional and turbulent jets. For example, both Strange \& Crighton (1983) and Gaster et al. (1985) attribute departures of observed growth rates from linear theory to nonlinear wave interactions. Numerical simulations at low Reynolds number have led to similar conclusions (Mohseni, Colonius \& Freund 2002). However, in transitional and forced jets, initial amplitudes and/or instability growth rates are generally higher than those associated with the natural turbulent mean flow field. It appears that, in the fully turbulent case, the instabilities reach lower overall amplitudes that prevent, at least on average, any significant effect of wave-wave interactions on their amplitude. 
(a)

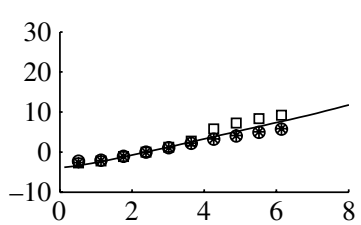

(b)

(c)

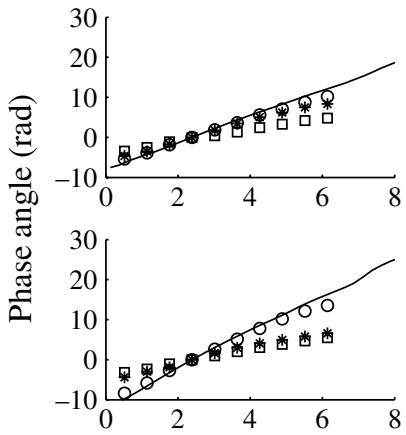

(d)

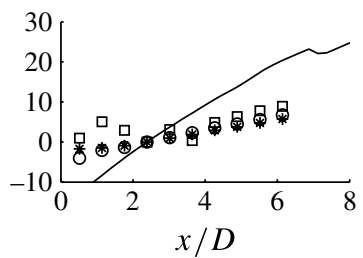

$m=1$
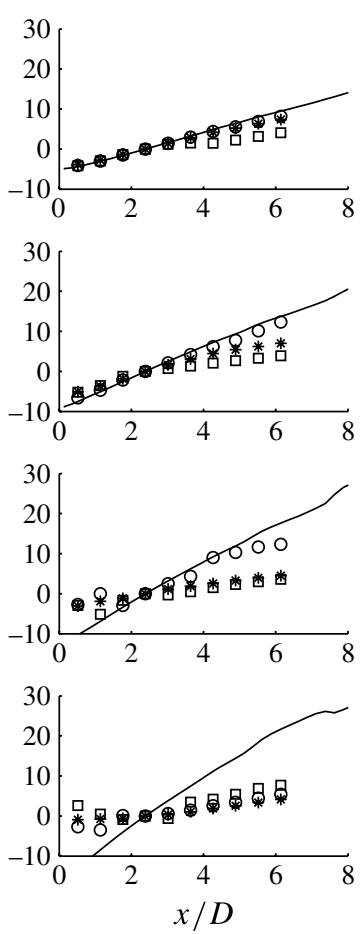

$m=2$
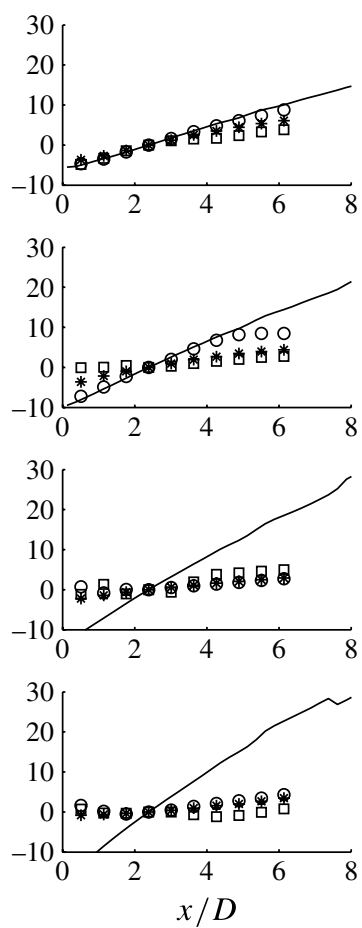

FIGURE 23. Phase along the microphone array for the hot $M_{\infty}=0.5$ jet: ensemble average $(*)$, first (o) and second ( $\square$ ) POD modes and PSE predictions (-), at frequencies of $S t=0.20(a), 0.35(b), 0.5(c)$ and $0.65(d)$.

We do not, however, believe that our conclusion suggests that the dynamics of the large-scale structures are linear in a deterministic, instantaneous sense. Nonlinearity is fundamental in establishing the turbulence cascade and the Reynolds stresses that give rise to the mean flow field that we use as the basic flow in our analysis. Nonlinearity is therefore implicitly, and partially, already accounted for. In all our comparisons, we are looking at statistically averaged data. In any particular realization, we can still find structures that are at least qualitatively similar to the averaged ones; but in general it is only the averaged structures that give rise to quantitative agreement with the PSE. There is significant jitter associated with the receptivity process in natural jets, which leads to different phasing of the different frequencies and azimuthal modes in any finite-time realization of the flow. If, over some time window, an instability wave at a particular frequency attains a higher-than-average amplitude, it will lead to a larger Reynolds stresses and a higher spread rate. New wavepackets of the same frequency will thereafter have smaller amplification, leading to a natural equilibrium with particular amplitudes associated with the long-time-averaged mean flow field.

This view is not new, essentially originating with Malkus' 'marginal theory of turbulence' (Malkus 1956). We believe, however, that the present analysis and data processing techniques are the first to show the process clearly in natural, highReynolds-number turbulent jet data, and to result in a rather consistent match between linear theory and measurements. In future, time-resolved PIV measurements, denser microphone arrays and, especially, simultaneous PIV and pressure measurements 
(a)

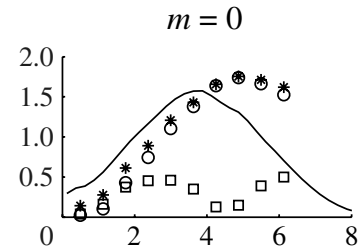

(b)

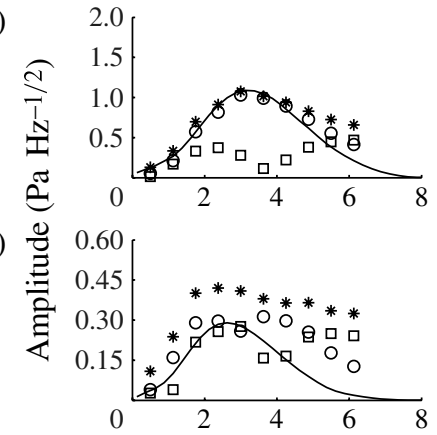

(d)

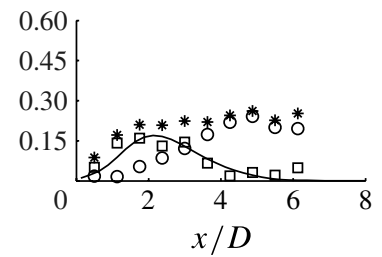

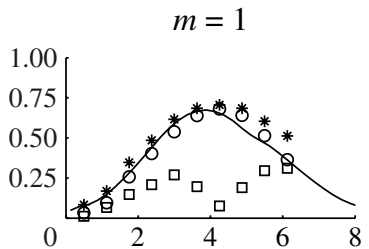
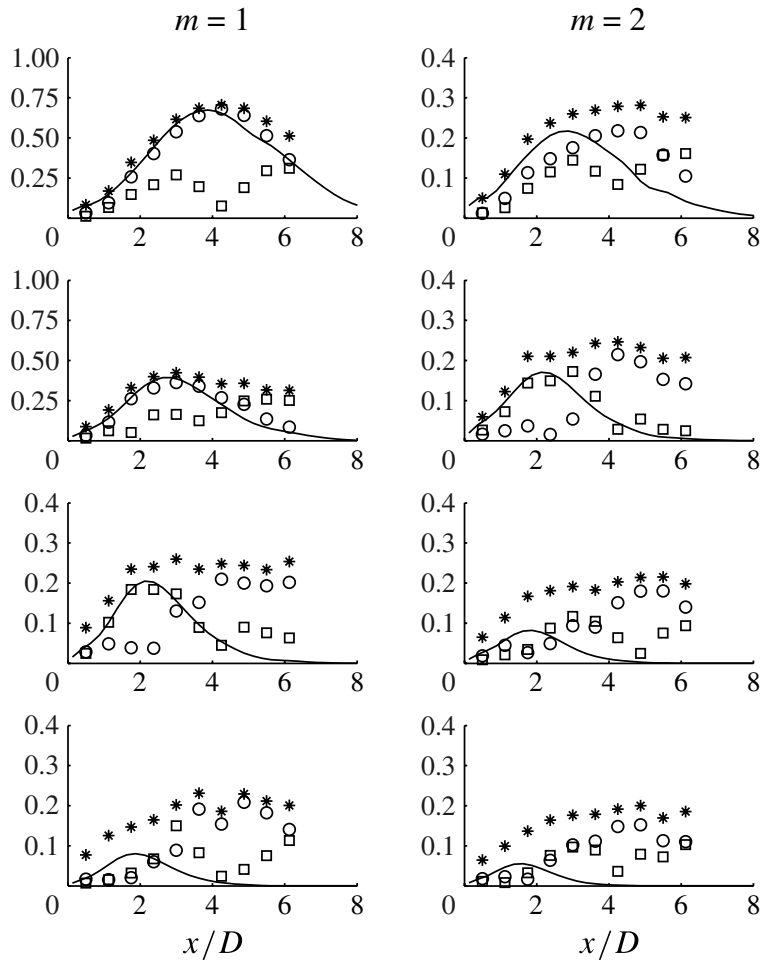
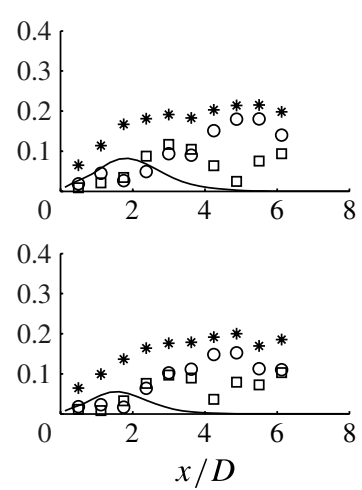

FIgURE 24. Pressure amplitude along the microphone array for the hot $M_{\infty}=0.9$ jet: r.m.s. data $(*)$, first $(\circ)$ and second $(\square)$ POD modes and PSE predictions (-), at frequencies of $S t=0.20(a), 0.35(b), 0.5(c)$ and $0.65(d)$. PSE amplitudes are based on first POD mode at $S t=0.25$ and 0.35 , and the second POD mode there above.

should permit more detailed identification of large-scale instability wave components in turbulent jets.

Finally, we point out that similar microphone array configurations have been used to investigate the extent to which the wavepacket structures in the near field give rise to the observed, aft-angle peak noise emission from turbulent jets (Colonius et al. 2010; Reba et al. 2010; Rodriguez et al. 2011). These studies suggest a viable alternative to Lighthill's acoustic analogy approach (Lighthill 1952) to predicting jet noise from large-scale structures, wherein linear disturbance equations such as PSE are used with mean flow field predictions from Reynolds-averaged turbulence models to predict the near acoustic field, and the linear wave equation is used then to extend the solution to the far field. The computational expense of such an approach is far less than corresponding large eddy simulations, and may be particularly useful in the analysis and control of jet noise.

\section{Acknowledgements}

The authors wish to thank Professor F. Hussain and Drs D. Rodriguez, A. Samanta and T. Suzuki for fruitful discussions on stability analysis and turbulence. We would also like to express appreciation to Dr J. Bridges and colleagues at the NASA Glenn Research Center for providing us with their data. This work was supported by the 
(a)

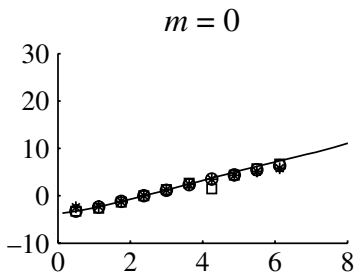

(b)

(c)

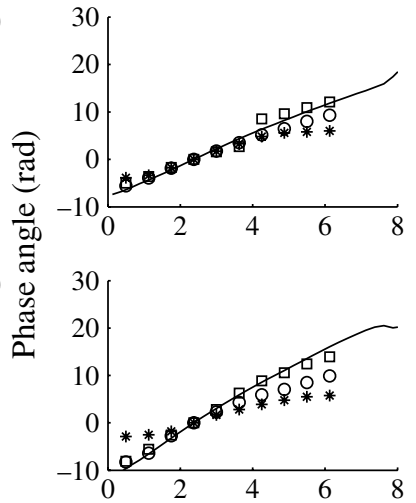

$(d)$

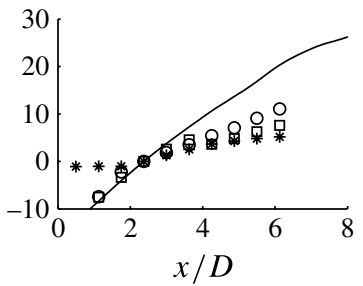

$m=1$
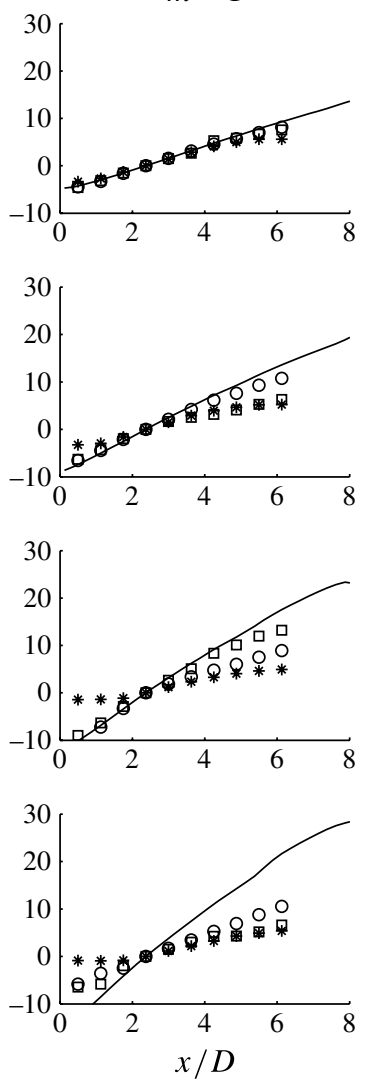

$m=2$
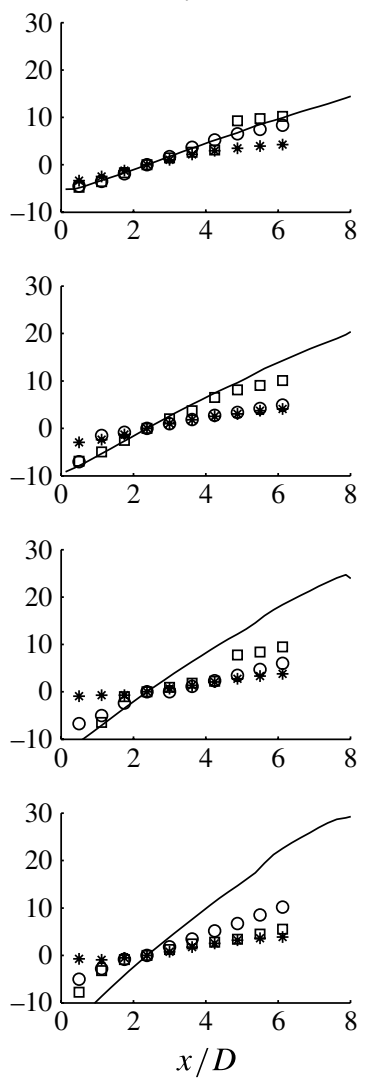

FIGURE 25. Phase along the microphone array for the hot $M_{\infty}=0.9$ jet: ensemble average $(*)$, first (o) and second ( $\square)$ POD modes and PSE predictions (-), at frequencies of $S t=0.20(a), 0.35(b), 0.5(c)$ and $0.65(d)$.

Aeroacoustics Research Consortium and TTC Technologies under an SBIR grant from NAVAIR, with Dr J. Spyropolous as technical monitor.

\section{Appendix. A higher frequency of the higher-speed and heated jets}

Here we show, in figures 20-25, further comparisons between the PSE predictions, the unfiltered microphone data and POD modes, for the cold $M_{\infty}=0.9$ jet and the hot $M_{\infty}=0.5$ and $M_{\infty}=0.9$ jets. These comparisons are made at frequencies $S t=0.20$, $0.35,0.5$ and 0.65 . See $\S 4.2$ for further discussion.

\section{REFERENCES}

ARndt, R. E. A., Long, D. \& Glauser, M. 1997 The proper orthogonal decomposition of pressure fluctuations surrounding a turbulent jet. J. Fluid Mech. 340, 1-33.

Balakumar, P. 1998 Prediction of supersonic jet noise. AIAA Paper 1998-1057.

Bertolotti, F. P. \& Herbert, T. 1991 Analysis of the linear stability of compressible boundary layers using the PSE. Theor. Comput. Fluid Dyn. 3, 117-124. 
Bertolotti, F. P., Herbert, T. \& Spalart, P. R. 1992 Linear and nonlinear stability of the Blasius boundary layer. J. Fluid Mech. 242, 441-474.

BRIDGES, J. \& WERnET, M. P. 2003 Measurements of the aeroacoustic sound source in hot jets. AIAA Paper 2003-3130.

Brown, G. L. \& Roshкo, A. 1974 On density effects and large structure in turbulent mixing layers. J. Fluid Mech. 64, 775-816.

Chang, C. L., Malik, M. R., Erlebacher, G. \& Hussaini, M. Y. 1993 Linear and nonlinear PSE for compressible boundary layers. Tech. Rep. ICASE 93-70.

Cheung, L. \& Lele, S. 2009 Linear and nonlinear processes in two-dimensional mixing layer dynamics and sound radiation. J. Fluid Mech. 625, 321-351.

Chomaz, J. 2005 Global instabilities in spatially developing flows: non-normality and nonlinearity. Annu. Rev. Fluid Mech. 37 (1), 357-392.

Cohen, J. \& Wygnanski, I. 1987 The evolution of instabilities in the axisymmetric jet. Part 1. The linear growth of disturbances near the nozzle. J. Fluid Mech. 176, 191-219.

Colonius, T., Samanta, A. \& Gudmundsson, K. 2010 Parabolized stability equation models of large-scale jet mixing noise. Procedia Engng 6, 64-73.

Crighton, D. G. \& Gaster, M. 1976 Stability of slowly diverging jet flow. J. Fluid Mech. 77, 397-413.

Crow, S. C. \& Champagne, F. H. 1971 Orderly structure in jet turbulence. J. Fluid Mech. 48, $547-591$.

Day, M. J., Mansour, N. N. \& ReYnolds, W. C. 2001 Nonlinear stability and structure of compressible reacting mixing layers. J. Fluid Mech. 446, 375-408.

Fleury, V., BAIlly, C. \& JuvE, D. 2005 Shear-layer acoustic radiation in an excited subsonic jet: models for vortex pairing and superdirective noise. C. R. Méc. 333 (10), 754-761.

GAster, M., Kit, E. \& Wygnanski, I. 1985 Large-scale structures in a forced turbulent mixing layer. J. Fluid Mech. 150, 23-39.

George, W. K., Beuther, P D \& ARndt, R. E. A. 1984 Pressure spectra in turbulent free shear flows. J. Fluid Mech. 148, 155-191.

GUDMUNDSSON, K. 2010 Instability wave models of turbulent jets from round and serrated nozzles. $\mathrm{PhD}$ thesis, California Institute of Technology.

Gudmundsson, K. \& Colonius, T. 2009 Parabolized stability equation models for turbulent jets and their radiated sound. AIAA Paper 2009-3380.

Herbert, T. 1997 Parabolized stability equations. Annu. Rev. Fluid Mech. 29, 245-283.

Huerre, P. \& Crighton, D. G. 1983 Sound generation by instability waves in a low Mach number jet. AIAA Paper 1983-0661.

Hussain, A. K. M. F. \& Reynolds, W. C. 1970 The mechanics of an organized wave in turbulent shear flow. J. Fluid Mech. 41, 241-258.

LAufer, J. \& Yen, T. C. 1983 Noise generation by a low-mach-number jet. J. Fluid Mech. 134, $1-31$.

Lesshafft, L. \& Huerre, P. 2007 Linear impulse response in hot round jets. Phys. Fluids 19, 024102.

LI, F. \& MALIK, M. R. 1996 On the nature of PSE approximation. Theor. Comput. Fluid Dyn. 8, $253-273$.

LI, F. \& MALiK, M. R. 1997 Spectral analysis of parabolized stability equations. Comput. Fluids 26, $279-297$.

Lighthill, M. J. 1952 On sound generated aerodynamically. I. General theory. Proc. R. Soc. Lond. A 211 (1107), 564-587.

Lumley, J. L. 1967 The structure of inhomogeneous turbulent flows. In Atmospheric Turbulence and Radio Wave Propagation (ed. A. M. Yaglom \& V. I. Tatarski). pp. 116-178. Nauka.

Maestrello, L. \& Fung, Y. T. 1979 Quasi-periodic structure of a turbulent jet. J. Sound Vib. 64, $107-122$.

Malik, M. R. \& Chang, C. L. 2000 Non-parallel and nonlinear stability of supersonic jet flow. Comput. Fluids 29, 327-365. 
Malkus, W. V. R. 1956 Outline of a theory of turbulent shear flow. J. Fluid Mech. 1 (5), 521-539.

MANKBADI, R. R. 1985 On the interaction between fundamental and subharmonic instability waves in a turbulent round jet. J. Fluid Mech. 160, 385-419.

MANKBADI, R. \& LiU, J. T. C. 1981 A study of the interactions between large-scale coherent structures and fine-grained turbulence in a round jet. Phil. Trans. R. Soc. Lond. A 298 (1443), 541-602.

Mattingly, G. E. \& ChAng, C. C. 1974 Unstable waves on an axisymmetric jet column. J. Fluid Mech. 65, 541-560.

Michalke, A. \& FUCHS, H. V. 1975 On turbulence and noise of an axisymmetric shear flow. J. Fluid Mech. 70, 179-205.

Mohseni, K., Colonius, T. \& Freund, J. B. 2002 An evaluation of linear instability waves as sources of sound in a supersonic turbulent jet. Phys. Fluids 14, 3593.

MOLlo-Christensen, E. 1967 Jet noise and shear flow instability seen from an experimenter's viewpoint. J. Appl. Mech. 34, 1-7.

Monkewitz, P. A. \& Sohn, K. D. 1988 Absolute instability in hot jets. AIAA J. 26 911-916.

Moore, C. J. 1977 The role of shear-layer instability waves in jet exhaust noise. J. Fluid Mech. 80, 321-367.

Morris, P. J., Giridharan, M. G. \& Lilley, G. M. 1990 On the turbulent mixing of compressible free shear layers. Proc. R. Soc. Lond. A 431 (1882), 219-243.

Petersen, R. A. \& SAmet, M. M. 1988 On the preferred mode of jet instability. J. Fluid Mech. 194, 153-173.

Pinier, J. T., Hall, J. W. \& Glauser, M. N. 2006 Identifying the most energetic modes of the pressure near-field region of a Mach 0.85 axisymmetric jet. AIAA Paper 2006-314.

Piot, E., Casalis, G., Muller, F. \& Bailly, C. 2006 Investigation of the PSE approach for subsonic and supersonic hot jets. Detailed comparisons with LES and linearized Euler equations results. Intl J. Aeroacoust. 5, 361-393.

Ray, P. K., Cheung, L. C. \& Lele, S. K. 2009 On the growth and propagation of linear instability waves in compressible turbulent jets. Phys. Fluids 21, 054106.

Reba, R., NARAyanan, S. \& Colonius, T. 2010 Wave-packet models for large-scale mixing noise. Intl J. Aeroacoust. 9, 533-558.

Rodriguez, D., Samanta, A., Cavalieri, A. V. G. \& Colonius, T. 2011 Parabolized stability equation models for predicting large-scale mixing noise of turbulent round jets. AIAA Paper, 2838.

Strange, P. J. R. \& Crighton, D. G. 1983 Spinning modes on axisymmetric jets. Part 1. J. Fluid Mech. 134, 231-245.

SuzuKi, T. \& Colonius, T. 2006 Instability waves in a subsonic round jet detected using a near-field phased microphone array. J. Fluid Mech. 565, 197-226.

TAM, C. K. W. \& Burton, D. E. 1984 Sound generated by instability waves of supersonic flows. Part 2. Axisymmetric jets. J. Fluid Mech. 138, 273-295.

TAM, C. K. W. \& Morris, P. J. 1985 Tone excited jets. Part V. A theoretical model and comparison with experiment. J. Sound Vib. 102 (1), 119-151.

TANnA, H. K. 1977 An experimental study of jet noise. Part I. Turbulent mixing noise. J. Sound Vib. 50, 405-428.

Tanna, H. K. \& Ahuja, K. K. 1985 Tone excited jets. Part I. Introduction. J. Sound Vib. 102 (1), $57-61$.

Thompson, K. W. 1987 Time dependent boundary conditions for hyperbolic systems. J. Comput. Phys. 68, 1-24.

Tinney, C. \& Jordan, P. 2008 The near pressure field of co-axial subsonic jets. J. Fluid Mech. 611, 175-204.

Troutt, T. R. \& McLaughlin, D. K. 1982 Experiments on the flow and acoustic properties of a moderate-Reynolds-number supersonic jet. J. Fluid Mech. 116, 123-156.

WinANT, C. D. \& BRowAND, F. K. 1974 Vortex pairing: the mechanism of turbulent mixing-layer growth at moderate Reynolds number. J. Fluid Mech. 63, 237-255. 
Yen, C. C. \& Messersmith, N. L. 1998 Application of parabolized stability equations to the prediction of jet instabilities. AIAA J. 36, 1541-1544.

Zaman, K B M Q \& Hussain, A. K. M. F. 1980 Vortex pairing in a circular jet under controlled excitation. Part 1. General jet response. J. Fluid Mech. 101, 449-491. 